\title{
Europa Explorer-An Exceptional Mission Using Existing Technology
}

\author{
Karla B. Clark \\ Jet Propulsion Laboratory \\ California Institute of Technology \\ 4800 Oak Grove Drive, Mail Stop 301-360 \\ Pasadena, CA 91109-8099 \\ (818) 354-9033 \\ karla.b.clark@jpl.nasa.gov
}

\begin{abstract}
A mission to Europa has been identified as a high priority by the science community for several years. The difficulty of an orbital mission - due primarily to the propulsive requirements and Jupiter's trapped radiationled to many studies that investigated various approaches to meeting the science goals. The Europa Orbiter Mission studied in the late 1990s only met the most fundamental science objectives. The science objectives have evolved with the discoveries from the Galileo mission. JPL studied one concept-Europa Explorer-for a Europa orbiting mission that could meet a much-expanded set of science objectives. A study science group was formed to verify that the science objectives and goals were being adequately met by the resulting mission design concept. The Europa Explorer design emerged primarily from two key selfimposed constraints:
\end{abstract}

1) Meet the full set of identified non-lander science objectives.

2) Use only existing technology.

Though other design concepts are viable for alternate program constraint sets, this paper will only discuss the results of this concept study. ${ }^{12}$

The Europa Explorer conducts a multi-year study of the Jupiter system, relies on existing technologies, and returns considerably more science data than previously examined conventional-propulsion mission concepts. In this mission concept, a single orbiter would traverse to Jupiter by means of a gravity assist trajectory and reach Jupiter $\sim 6$ years after launch, followed by an $\sim 1 \frac{1}{2}$ year tour of the Galilean satellites, before entering Europa orbit. The Europa Prime Mission would be 90 days, with the expectation of being operational for upwards of a year. The flight system would return approximately three year's worth of Cassini data $(\sim 3$ Tbit) during the first 90 days in Europa orbit. During this Europa Prime Mission, the flight system would orbit Europa more than 1000 times and provide three orders of magnitude more close $(<5000-\mathrm{km}$ altitude) Europa observing time than Galileo.

\footnotetext{
${ }^{1} 1-4244-0525-4 / 07 / \$ 20.00$ C2007 IEEE

2 IEEEAC paper \#1417, Version 6, Updated July 1, 2006
}

The initial portion of this study resulted in a highly robust science mission as summarized above. Subsequent discussions with the Outer Planets Assessment Group (OPAG, November 2006) led to the definition of a more modest reference mission. This reference mission is also described.

\section{TABLE OF CONTENTS}

$\begin{array}{lr}\text { 1.0 INTRODUCTION } & 1 \\ \text { 2.0 STUDY DEFINITION } & 2 \\ \text { 3.0 INITIAL DESIGN CONCEPT } & 4 \\ \text { 4.0 TECHNICAL DISCUSSION } & 9 \\ \text { 5.0 OPEN ISSUES } & 17 \\ \text { 6.0 REFERENCE MISSION DEFINITION } & 18 \\ \text { 7.0 CONCLUSIONS } & 18 \\ \text { REFERENCES } & 16 \\ \text { BIOGRAPHY } & 20 \\ \text { ACKNOWLEDGEMENTS } & 20\end{array}$

\subsection{INTRODUCTION}

Europa, Jupiter's second Galilean satellite, is among the most interesting targets for planetary exploration in the solar system. Mission studies have investigated many of the challenges associated with exploring Europa. Europa orbital concepts can satisfy a significant number of the science objectives, but require extensive propulsion systems to achieve orbit. Another option-flybys mission conceptsmight have a more limited science appeal. Radiation levels near Europa require detailed design-mitigation approaches and will highly influence any mission lifetime. Designing any mission to Europa requires a complex trade of technical, programmatic, and scientific parameters; to date, these trades have not yet been sufficiently explored.

The National Research Council's (NRC) Decadal Survey of Solar System Exploration [1] ranked a Europa orbital mission as the highest-priority large ("flagship") mission for solar system exploration in the near term. The NRC's Committee on Planetary and Lunar Exploration (COMPLEX) concluded that Europa exploration should be assigned a priority equal to that of Mars Exploration [2]. Numerous other advisory bodies, including NASA's Solar System Exploration Committee and NASA's recent Roadmap Committee for Solar System Exploration, have 
made strong recommendations to initiate as soon as possible the next stage of Europa exploration, including orbital studies and, potentially, landed payloads.

All of these scientific studies have highlighted the highpriority scientific objectives required to make major advances in our current understanding of Europa: confirmation of an ocean, study of the ice crustal structure, geologic history of exchange between the ocean and surface, and the chemical composition of the non-water materials on the surface, including organics, if present.

Post-Galileo exploration of Europa presents a number of major technical challenges. Accomplishing the large number of the science objectives addressed by Europa scientists requires a more complex mission than a repeat of Voyager or Galileo-style flybys. In turn, this translates to a requirement to not only get into Jupiter orbit, but to orbit Europa itself and survive and operate within Jupiter's trapped radiation environment long enough to achieve the major objectives.

In the last ten years, three major mission concepts for Europa exploration from Europa orbit have been studied:

1) Europa Orbiter (EO) was cancelled in 2001 (Phase B) due to a combination of the perceived lack of readiness of radiation-tolerant electronics, limited scientific payload capability $(\sim 25 \mathrm{~kg})$, and because this concept offer only a brief data-taking period ( $\sim 30$ days in orbit).

2) Jupiter Icy Moons Orbiter (JIMO), the first mission of the Prometheus Program, involved development of nuclear-electric propulsion to orbit each of the icy Galilean satellites, ending with Europa. Significantly more ambitious and scientifically capable than EO, JIMO was indefinitely deferred in 2005 (Phase A), primarily due to the large initial investment in space nuclear propulsion infrastructure required, combined with NASA's emphasis on human exploration of the Moon and Mars.

3) Europa Geophysical Explorer (EGE), a mission similar to the Europa Orbiter, was studied during the Summer of 2005 (Pre-Phase A). This mission concept took advantage of technology advancements and updated Level-1 requirements from NASA Headquarters.

The investments in technology and research for these past mission concepts, particularly in the areas of radiation tolerant electronics and complex mission design, have now put NASA in a position to develop a Europa exploration concept in the flagship mission class that relies on demonstrated technologies and achieves the high-level science objectives. JPL initiated a study to determine specific solutions to the major challenges associated with Europa exploration. There exist a wide range of Europa mission concepts, including flybys, impactors, landers, orbiters, and sub-satellites that have varying science value with implementation. This study focused on the option that was considered to address a vast set of science objectives while not overly stressing technology or funding requirements. Thus, it is only one point in the much larger trade space that should be evaluated.

The main focus of the study was to explore the possibility of a Europa orbiting mission that addressed a significant number of science objectives while using current technology. The mission concept was worked at length with a Study Science Team comprising several Europa Subgroup (ESG) scientists of the Outer Planets Assessment Group (OPAG), which is led by Dr. Ronald Greeley of Arizona State University. The Science Study Team relied on the wide-ranging development of Europa science objectives by previous studies and advisory committees.

The three-month study focused on answering critical challenges that were identified prior to embarking on the effort. JPL had extensively studied other mission concepts for Europa over the nine previous years. As these missions were studied, key areas of emphasis became apparent: science orbit achievability, uncertain knowledge of the radiation environment, solar power source technology maturity, science data return, and radiation hardened electronics capability. Small focused teams were identified and charged with investigating each of these issues relative to the current state of knowledge and technology maturity. Four teams were formed: Science Orbit Maintenance Team, Science Data Return Team, Solar Array Feasibility Team, and Implementation Assessment Team.

Landing on the potentially fractured surface of Europa is difficult and risky. Without an atmosphere, a soft landing becomes even more difficult due to the required propulsive descent system. Lander/impactors were not investigated here but should be studied in the future.

More recently, additional trade studies have been performed and discussed with the Science community to arrive at a lower mass (and expected cost) implementation which fully meets all the science objectives but is slightly less capable. The results of the initial study are described while the modifications required defining the reference mission are discussed in Section 6.0.

\subsection{STUDY DEFINITION}

A three-month Europa Explorer (EE) study was initiated to clearly identify critical challenges and to determine solutions to those challenges using today's technologies and approaches. The study drew on JPL's extensive Europa experience to identify and explore these critical technical issues relating to a Europa mission. Each critical issue was documented along with conclusions, open areas, and order of priority for areas requiring further investigation. The results of the study included not only the individual assessments of the challenges but an integrated Europa mission concept that was verified by the Study Science 
Team to ensure that the science goals and objectives were being adequately addressed by the mission design.

\section{Requirements and Objectives}

The study made use of the information from the NASAfunded EGE Study executed over the Summer of 2005 [3]. There was no additional information available to further update the Level-1 requirements, so this study used the Level-1 requirements that were available from the EGE study. Thus, the Level-1 requirements for this EE study are:

1) L/V: Delta IVH available

2) Primary Propulsion: Chemical

3) Power: RPS Power Systems acceptable

4) Orbital Mission: 30 days minimum to meet orbital science objectives

5) Earth gravity assists: Allowed

6) Planetary protection: Europa impact acceptable assuming appropriate measures for sterilization are employed

The EE science objectives were developed in collaboration with the Study Science Team. The Science Study Team was comprised of 13 scientists from Arizona State University, University of California Los Angeles, University of Arizona, University of Houston, University of Colorado, and JPL. The Science Study Team worked from the ESG objectives and re-affirmed that these objectives were appropriate for this study. The adopted science objectives for EE are traced to the two of the key questions in the NRC Space Studies Board's Decadal Survey [1]; namely:

1) What planetary processes are responsible for generating and sustaining habitable worlds, and where are the habitable zones in the solar system?

2) How do the processes that shape the contemporary character of planetary bodies operate and interact?

In response to these questions, the ESG established the following six science objectives for the study of Europa by the EE mission:

1) Confirm the presence of a subsurface ocean.

2) Characterize the three-dimensional configuration of the icy crust, including possible zones of liquid.

3) Map organic and inorganic surface compositions, especially as related to astrobiology.

4) Characterize surface features and identify candidate sites for future exploration.

5) Characterize the magnetic field and radiation environment.
6) Understand the heat source(s) and time history of Europa's ocean.

These objectives are quite similar to those adopted by the JIMO Science Definition Team (SDT) for Europa.

Specific measurement requirements were derived from the six science objectives; from these measurement requirements specific instrument requirements were defined. The measurement requirements were classified as Priority 1 through 6 by the ESG. Note that each objective has several measurement requirements. The EE Study Science Team took the ESG input and adopted it for the EE mission concept. The EE mission concept meets all the Priority 1 measurement requirements in 30 days.

\section{Guidelines}

Additional guidelines were used to further refine the mission concept. These guidelines were imposed to confine the effort to boundaries that were not overly stressing yet still allowed adequate flexibility in future trade spaces and mission design parameters. The guidelines are, as follows:

1) No new technology - For the purpose of this study, only technology developments that are funded, ongoing, and scheduled to be completed within calendar year 2007 were assumed. Technologies that would further significantly enhance the mission were evaluated and are discussed in the study reports.

2) High-data-rate science taken at Earth distances of 5.5 AU or less-This guideline allows for sizing of the data return path under reasonable conditions while imposing only minor constraints on tour design for timing of arrival at Europa. This is consistent with the desire to implement the mission away from superior conjunction.

3) 90-day Europa orbiting Prime Mission-Radiation exposure is the life-limiting characteristic of this mission. Thus, the design point for radiation becomes crucial. JPL has documented approaches to radiation design founded in experience and lessons learned that were followed for this study as well. The choice of mission duration was worked extensively with the Study Science Team to trade science data return volume with mass. Ultimately, the radiation requirement is to design to high confidence $(95 \%)$ of seeing a radiation dose below the design point at 3-month (90 days) and $50 \%$ chance at 7.5 months (225 days).

4) JPL Design Principles-JPL has developed a set of design principles that are imposed on all flight missions. These principles were developed over the course of many years using Lessons Learned and best practices from previous missions. The design resulting from this EE study meets or exceeds all Design, Verification/Validation and Operations Principles for Flight Systems (D-17868) [4]. 


\section{Science Derived Requirements}

The characteristics of the desired science orbit about Europa have been evaluated over the course of several years by members of the science community. The principal science drivers are identified as imaging, radar, and geodesy that push for low-altitude orbits with extremely tight orbital knowledge. The EO SDT and, subsequently, the JIMO SDT spent many months evaluating the science orbit parameters and reconciling the multiple scientific constraints. In the end, both groups adopted very similar requirements for the science orbit. The latest version of these requirements, as documented by the JIMO SDT, was adopted for this EE study:

1) Inclination $\geq 70^{\circ}$.

2) Eccentricity starting value near circular $(\sim 0.001)$.

3) Altitude 100 to $500 \mathrm{~km}$.

4) Nodal phase angles $30^{\circ}$ to $70^{\circ}$.

To achieve the Europa geodesy objectives connected with confirming the presence of a subsurface ocean, the spacecraft orbit must be reconstructed to an accuracy of $1 \mathrm{~m}$ in the radial direction. To achieve this level of accuracy, adequate levels of Doppler tracking (dual frequency is preferred) are required and $\Delta \mathrm{V}$ maneuvers must be restricted to less than one per day for at least the first few Europan days in orbit. The remote sensing portion of the payload needs to view in the nadir direction when in orbit about Europa.

Feasible total payload mass will be in the neighborhood of $\sim 180 \mathrm{~kg}$ for EE; total orbital average power available for science will be $\sim 100 \mathrm{~W}$. These numbers were derived originally from estimates of the instrument masses and shielding required for the planning payload with appropriate margins added.

\section{Radiation Environment Derived Requirements -}

The radiation design requirement for this mission study is driven by the Prime Mission duration of 90 days. The statistical Galileo Interim Radiation Electron (GIRE) model was used to determine the predicted mean radiation dose expected at 90 days in Europa orbit [5,6]. A Radiation Design Factor (RDF) is used as required by JPL Design Principles [4] and lessons learned from actual failure history of Galileo were incorporated $[7,8]$.

The radiation design requirement for Total Ionizing Dose (TID) is:

1) 3.4 Mrad Si environment behind 100 mils of Aluminum equivalent.

2) $\mathrm{A}$ RDF of 2 is used for all components on the spacecraft resulting in a design point of $6.8 \mathrm{Mrad} \mathrm{Si}$ behind 100 mils of Aluminum equivalent.
3) A RDF of 3 is required for all spot shielding including electronic parts with a radiation tolerance below 150 $\mathrm{krad}$ which results in a design point of $10.2 \mathrm{Mrad} \mathrm{Si}$ behind 100 mils of Aluminum equivalent.

4) Exposed surfaces design to their expected environment behind their actual shielding plus a RDF of 2 .

The statistical GIRE model determines the expected doses for different radiation species. Each of these species reacts slightly differently within electronics and materials. Thus the actual distribution of the radiation species within the TID requirement above is broken down in Figure 2-1 as a function of equivalent shield thickness. As shown in Figure $2-1$, the Jupiter environment is dominated by electrons except at very thin shield thicknesses $(<10$ mils) where protons are important and very thick shields ( $>1000$ mils) where photons and Gammas (from RTGs) begin to dominate.

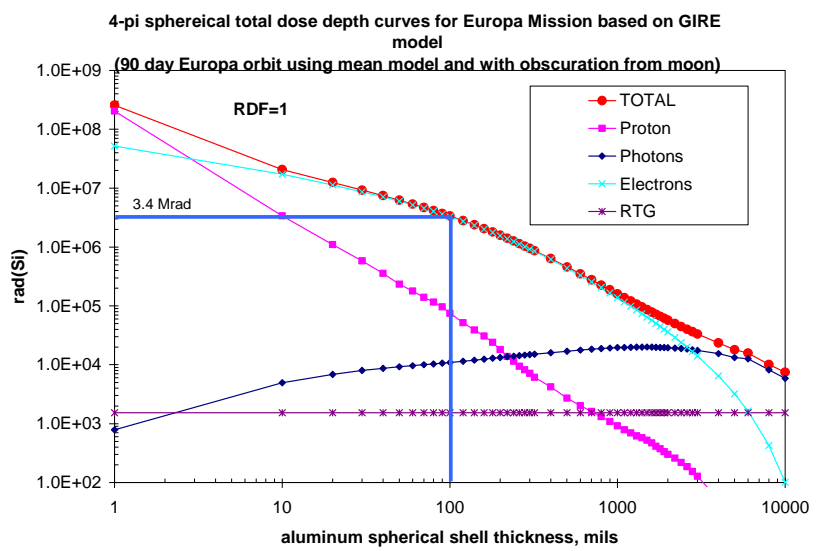

Figure 2-1 Dose Depth Curve for Selected Radiation Design Point

\subsection{INITIAL DESIGN CONCEPT}

The EE concept utilizes a chemically propelled spacecraft powered by Radioisotope Thermoelectric Generators to deliver 10 science instruments into orbit around Europa (see Figure 3-1 and Table 3-1).

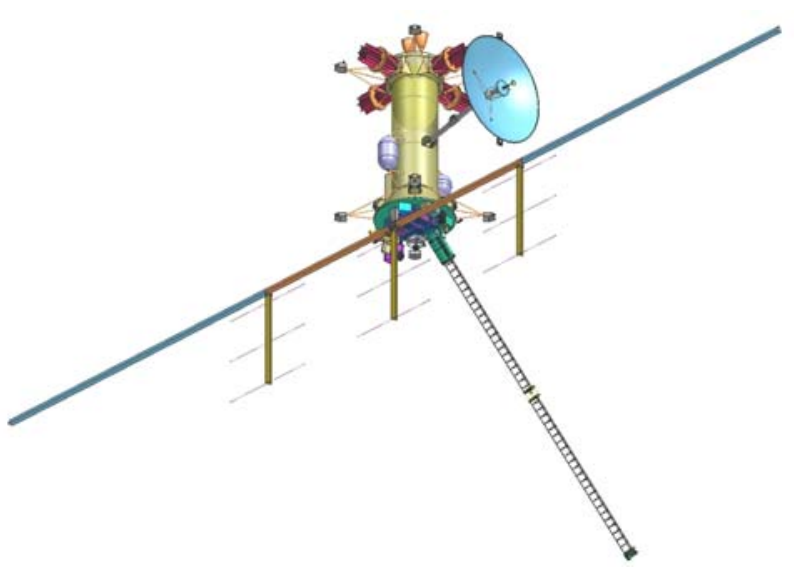

Figure 3-1 Spacecraft On-Orbit Configuration 
Table 3-1 Key Spacecraft Performance Parameters

\begin{tabular}{|c|c|c|}
\hline Parameter & Value & Notes \\
\hline \multicolumn{3}{|l|}{ Instruments } \\
\hline $\begin{array}{l}\text { Number of } \\
\text { instruments }\end{array}$ & 10 & $\begin{array}{l}\text { Does not include Ka-band } \\
\text { uplink/downlink equipment used for } \\
\text { gravity science that is included within } \\
\text { the telecom subsystem. }\end{array}$ \\
\hline Instrument mass & $182 \mathrm{~kg}$ & $\begin{array}{l}\text { Includes } 153 \mathrm{~kg} \text { for instruments with } \\
43 \% \text { contingency and } 29 \mathrm{~kg} \text { (CBE + } \\
\text { contingency) for shielding. Does not } \\
\text { include } 5.2 \mathrm{~kg} \text { (CBE) Ka-band } \\
\text { uplink/downlink equipment tracked in } \\
\text { telecom mass estimate. }\end{array}$ \\
\hline Instrument power & $143 \mathrm{~W}$ & $\begin{array}{l}\text { Average power use in orbit at Europa. } \\
\text { Includes } 43 \% \text { contingency. Does not } \\
\text { include power for Ka-band } \\
\text { uplink/downlink equipment. }\end{array}$ \\
\hline \multicolumn{3}{|c|}{ Science Accommodation } \\
\hline Pointing accuracy & $\begin{array}{l}5 \text { mrads } \\
\text { (3 sigma) }\end{array}$ & $\begin{array}{l}\text { S/C body pointing control accuracy } \\
\text { during nadir-oriented non-thrusting } \\
\text { orbital period. }\end{array}$ \\
\hline Pointing stability & $\begin{array}{l}1 \mathrm{mrad} / \mathrm{s} \\
\text { (3 sigma) }\end{array}$ & $\begin{array}{l}\text { For body-fixed instruments in science } \\
\text { orbit during non-thrusting periods. }\end{array}$ \\
\hline $\begin{array}{l}\text { Minimum duration } \\
\text { between reaction } \\
\text { wheel orbit } \\
\text { desaturations }\end{array}$ & 24 hours & $\begin{array}{l}\text { Minimum duration between } \\
\text { desaturation thruster firings. }\end{array}$ \\
\hline Data storage & $\begin{array}{l}750 / 500 \\
\text { Mbits }\end{array}$ & $\begin{array}{l}\text { Science Data Recorder includes } 300 \\
\text { Mbits for science data, and } 450 \text { mbits } \\
\text { for instrument software. Flight value } \\
\text { includes storage for flight software } \\
\text { and telemetry. }\end{array}$ \\
\hline $\begin{array}{l}\text { Data rate } \\
\text { (average/max) }\end{array}$ & $\begin{array}{l}400 \mathrm{kbps} / 2 \\
\text { Mbps }\end{array}$ & $\begin{array}{l}\text { Average rate assumes range of } 5.5 \\
\mathrm{AU}, 3 \mathrm{~dB} \text { link margin, multiple data } \\
\text { rates optimized for elevation, Jupiter } \\
\text { presence, and specific stations during } \\
\text { the first } 30 \text { days in orbit. } \\
\text { Max rate assumes best case } \\
\text { conditions - range of } 4.1 \mathrm{AU}, 0 \mathrm{~dB} \text { link } \\
\text { margin, Jupier out of beam. } \\
\text { Both conditions assume } 90 \% \text { weather. }\end{array}$ \\
\hline \multicolumn{3}{|l|}{ Spacecraft } \\
\hline Processor speed & $132 \mathrm{MHz}$ & $\begin{array}{l}\text { Applies to science and flight } \\
\text { computers. }\end{array}$ \\
\hline $\begin{array}{l}\text { Available power at } \\
\text { EOM }\end{array}$ & $823 \mathrm{~W}$ & $\begin{array}{l}\text { Power output from } 8 \text { MMRTGs after } \\
8.5 \text { years, Battery for load-leveling }\end{array}$ \\
\hline $\begin{array}{l}\text { Main engine thrust } \\
\text { level }\end{array}$ & $900 \mathrm{~N}$ & $\begin{array}{l}\text { Two 900-N engines included (one } \\
\text { prime and one spare), } ~ 325 \text { ISP }\end{array}$ \\
\hline Delta V capability & $2608 \mathrm{~m} / \mathrm{s}$ & $\begin{array}{l}\text { Assuming launch mass is equal to the } \\
\text { launch vehicle capability }(7230 \mathrm{~kg}) \text {. }\end{array}$ \\
\hline Radiation tolerance & 3.4 Mrad & $\begin{array}{l}\text { Achieved with a Radiation Design } \\
\text { Factor (RDF) } \geq 2 \text {. }\end{array}$ \\
\hline $\begin{array}{l}\text { Heliocentric } \\
\text { operating range }\end{array}$ & $\begin{array}{c}0.66 \text { to } 5.5 \\
\text { AU }\end{array}$ & $\begin{array}{l}\text { Minimum range defined by VEEGA } \\
\text { trajectory. }\end{array}$ \\
\hline
\end{tabular}

The science instruments begin taking science once in the Jupiter system while the spacecraft uses Jupiter moon gravity assists to further reduce the energy required to enter Europa orbit. The orbital mission lifetime is ultimately limited by radiation dose accumulated once in Europa Orbit.

\section{Mission Description}

The mission design from launch through initial orbit at Europa is based on previous work, primarily for EO and EGE. No new trajectory work for those early phases of the mission was done for this three-month EE study. The primary enhancements to the EO concept baseline were interplanetary trajectories that now included gravity assists and a much more detailed analysis of the orbits at Europa. A significant amount of work regarding the orbital environment near Europa was accomplished as part of the JIMO development effort; the lessons learned from that work were applied and expanded as a part of this current study.

EO was limited to a direct trajectory (i.e., no planetary gravity assists) from Earth to Jupiter. Direct trajectories have a relatively short flight time (3 to 3.5 years), but require high launch energies, resulting in a relatively small injection mass capability for a given launch vehicle. Several gravity assist trajectory options were considered as part of the EGE and other studies. The types explored for the timeframe of interest were $\Delta \mathrm{V}$-Earth gravity-assist $(\Delta \mathrm{V}$ EGA) and Venus-Earth-Earth Gravity Assist (VEEGA) trajectories.

Other less characterized options are potentially available, including those with Mars gravity assist with and without Earth gravity assists. Using a Delta IVH launch vehicle (LV), the $\Delta$ V-EGA trajectories can deliver approximately twice the dry mass into Europa orbit as a direct trajectory and the VEEGA trajectories can deliver three or more times as much. Some of the 2015-16 trajectory options are shown in Figure 3-2.

A comparison of the dry mass capability and flight time for these trajectories is shown in Figure 3-3. The dry mass capability varies depending on the particular trajectory and launch day. For the VEEGA trajectories plotted, the dry launch mass varies from about $3150 \mathrm{~kg}$ to $3550 \mathrm{~kg}$.

The baseline trajectory used by the Implementation Assessment Team is a VEEGA departing Earth in June 2015 (Figure 3-4) that, assuming a Delta IVH launch vehicle, allows approximately $3000 \mathrm{~kg}$ to be delivered into Europa orbit after jettisoning the launch vehicle adapter (approximately $160 \mathrm{~kg}$ ).

On the initial approach to Jupiter, the trajectory utilizes a Ganymede flyby for a gravity assist prior to Jupiter orbit insertion (JOI). JOI occurs near perijove at around 12.5 Jovian radii and results in an orbit period of about 200 days. Near apojove of the first orbit, a perijove raise maneuver is performed to set up the Jovian tour.

The Jovian tour includes a dozen or more gravity assists with the Galilean moons prior to the final approach to Europa. The tour is used to reduce the energy of the orbit and the $\Delta \mathrm{V}$ needed to insert into orbit at Europa. There are a multitude of options available for the tour with different sequences of gravity assists. A tour typically lasts 0.5 to 1 year and requires little, if any, deterministic propellant. A representative Jovian tour is shown in Figure 3-5. 


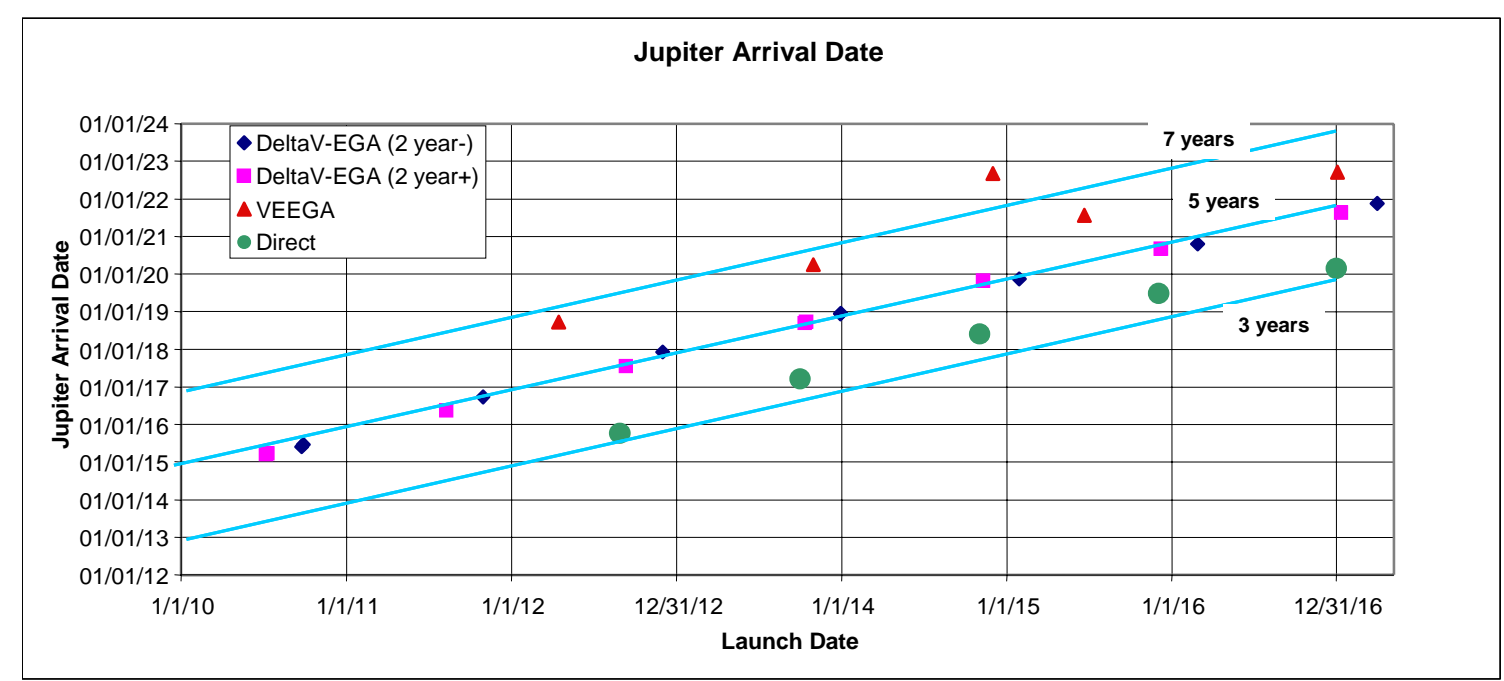

Figure 3-2 Jupiter Arrival Date vs Launch Date for Several Trajectory Options

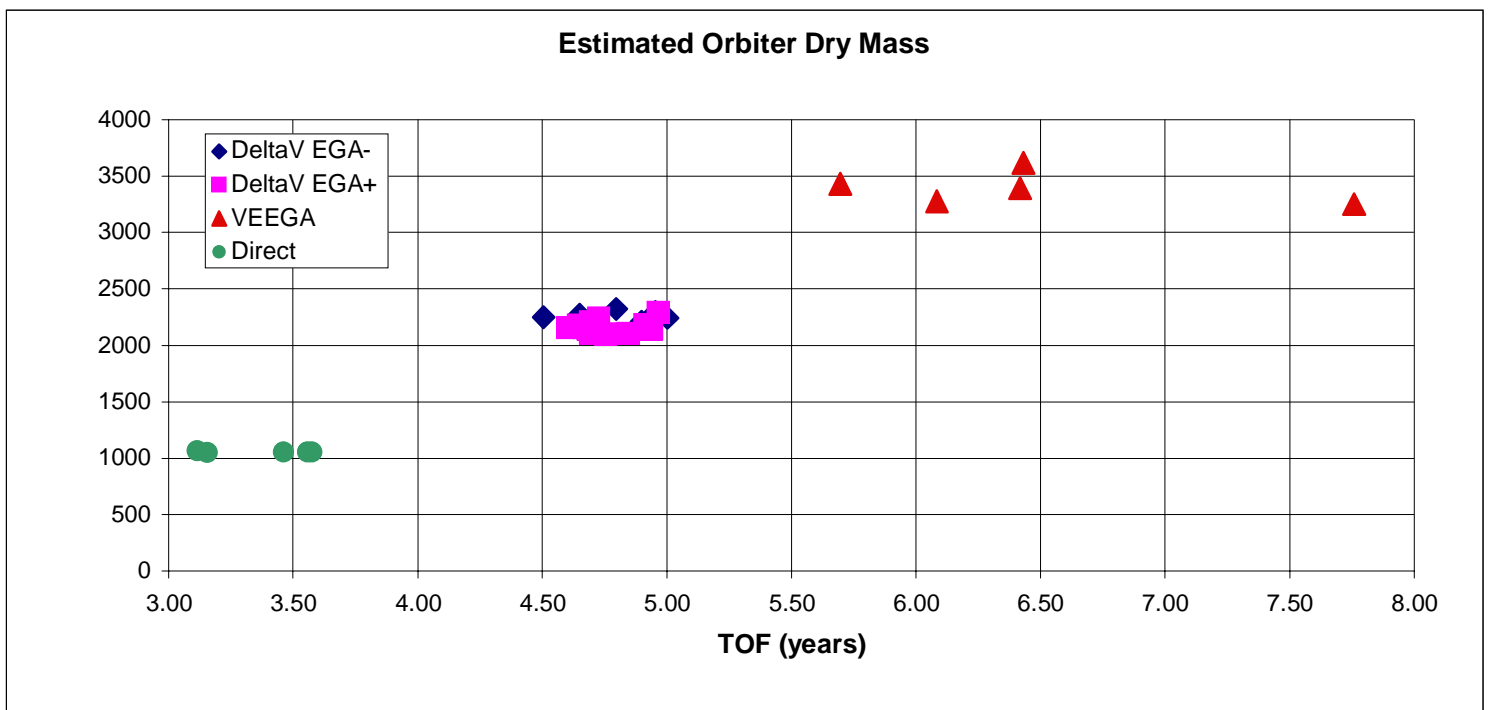

Figure 3-3 Dry Mass Capability vs. Flight Time for Several Trajectory Options (Delta IVH LV)

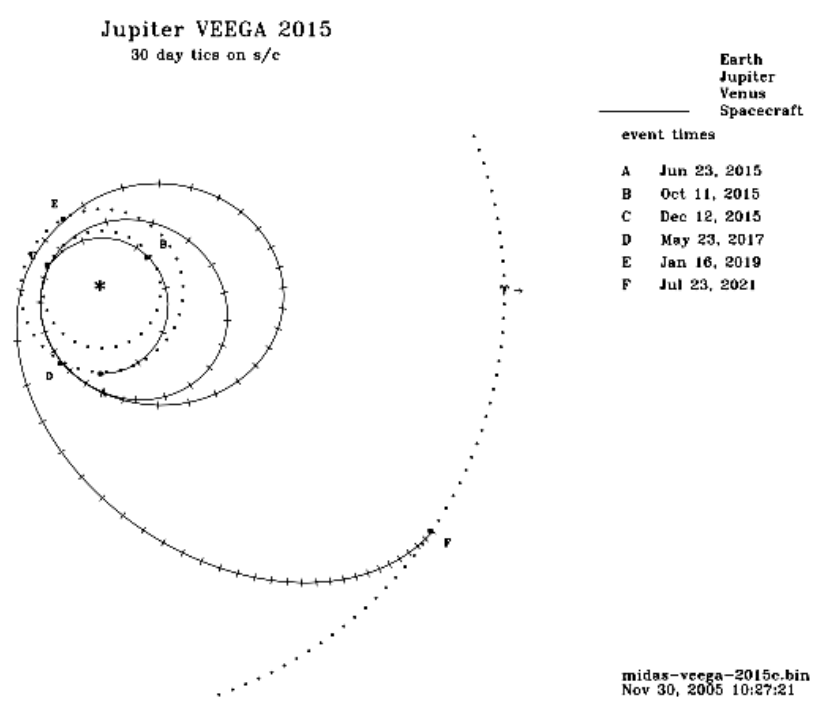

Figure 3-4 Example VEEGA Trajectory Used as Baseline for Implementation Assessment Team

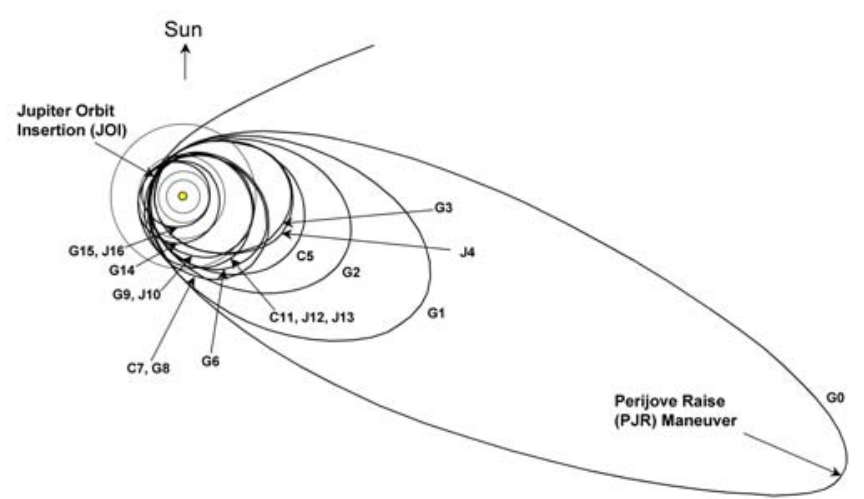

Figure 3-5 Example Jovian Tour Used as Baseline for Implementation Assessment Team 
The guideline that high-data-rate science would be taken at Earth distances of 5.5 AU or less might require a relatively longer flight time for the tour in order to set up the correct phasing for the approach to Europa; however, the large number of options available should otherwise alleviate the impact of this constraint.

The Europa flyby following the final Ganymede gravity assist sets up a near-resonant orbit with Europa. A maneuver is performed near apojove of that orbit followed by another Europa flyby setting up a lower energy near-resonant orbit. A maneuver is again performed near apojove, and Europa orbit insertion (EOI) occurs on the next approach to Europa. The latest EO concept used a 3:4 resonance followed by a 5:6 resonance; this entire final approach phase lasts about 45 days.

Other types of Europa approaches are possible and should be explored more fully. This final approach takes place within a high radiation environment; flight time for this phase is, therefore, a key characteristic that can be traded with $\Delta \mathrm{V}$ (propellant mass) to result in an optimal combination for the mission. Several innovative techniques for designing captures at Europa were developed as part of the JIMO work and should be analyzed for applicability to a relatively high-thrust (chemical propulsion) mission as conceived for the current study.

To satisfy the science objectives, the science orbit at Europa needs to be low altitude (100 to $500 \mathrm{~km}$ ), near circular, and near polar (within $20^{\circ}$ ). If left uncontrolled, orbits with these characteristics become more eccentric, due primarily to the gravitational effect of Jupiter and impact generally within a few tens of days. These orbits need to be maintained on a regular basis. There are very special cases of "frozen orbits" that have a relatively long lifetime, but the exact orbital conditions for these orbits depend on the details of the gravity field, which will not be known until the spacecraft has been in Europa orbit for several days and the science measurements can be made and analyzed. Even with frozen orbits, the semi-major axis and inclination have periodic variations of a few kilometers and a couple degrees, respectively.

The selection of a science orbit, an initial orbit at Europa, and transfer from an initial orbit to a science orbit is open to broad trades. It is unclear at this point whether the nominal science orbit should be a frozen orbit or not. If a frozen orbit is chosen, the orbit elements and characteristics would not be determined until after Europa arrival, and it would then take additional time to transfer to a frozen orbit. This needs to be further explored to understand instrument and operational impacts of science orbit selection and timing.
The stability of the orbits has a direct effect on the science orbit maintenance and, hence, the orbit determination. A trade exists between the frequency and total $\Delta \mathrm{V}$ required for the maintenance maneuvers, with smaller, more frequent maneuvers potentially resulting in less $\Delta \mathrm{V}$ overall. Lower total $\Delta \mathrm{V}$ results in less total time interruption to the science; however, the more frequent maneuvers might significantly degrade the orbit determination. Therefore, the selection of the precise elements for the science orbits and the orbit maintenance strategy are still not determined.

Once the mission is completed, the spacecraft will be left in Europa orbit. It is anticipated that the orbit will degrade relatively quickly (with a few months) such that the spacecraft will eventually impact the surface of Europa. The planetary protection requirements for this mission are not finalized (note, however, that work had been done for both EO and JIMO). The working assumption is that the radiation environment will sterilize external surfaces and that a box-level sterilization program will be required to ensure that all planetary protection requirements are met.

\section{Payload Description}

A planning payload was utilized to allow the scientists and engineers to develop a complete mission concept that addressed the identified science objectives within a reasonable set of requirements and constraints. The payload enables engineers to understand what requirements are imposed by different payload elements. The actual payload would be the result of a selection process carried out by NASA. The planning payload selected for the EE study consists of a notional set of 6 remote-sensing and 4 fieldsand-particles instruments. Table 3-2 gives the estimated resource requirements for each instrument and for the total payload.

Twenty kilograms (plus margin) of common radiation shielding is allocated which assumes housing the electronics in a common packaging structure. The results in a total mass allocation for Science instruments of $182 \mathrm{~kg}$. Detailed design work in this area is needed for specific instruments as they are proposed. The allocated masses were assessed by the Study Science Team and the spacecraft team and were thought to be adequate.

The Europa gravity field will not be known until the spacecraft is in orbit. Therefore, the exact science orbital parameters will not be known until then. Thus, instruments will need to be designed to allow for some in-flight flexibility. The type and degree of flexibility will need to be worked in the future with the selected Science Team. A discussion of these required parameters will need to be available for the solicitation of the science instruments. 
Table 3-2 Planning Payload Resource Requirements

\begin{tabular}{|c|c|c|c|c|}
\hline Instrument & Mass $(\mathrm{kg})$ & $\begin{array}{c}\text { Power }(\mathrm{W}), \\
\text { Day/Night }\end{array}$ & $\begin{array}{c}\text { Approximate } \\
\text { Dimensions }(\mathrm{cm})\end{array}$ & $\begin{array}{c}\text { Field of } \\
\text { View (deg) }\end{array}$ \\
\hline Wide-angle Camera (WAC) & 3 & $3 / 1$ & $\begin{array}{l}15 \times 5 \times 5 \text { (optics) } \\
5 \times 15 \times 20 \text { (electronics) }\end{array}$ & $59 \times 59$ \\
\hline Medium-angle Camera (MAC) & 10 & $10 / 1$ & $\begin{array}{l}15 \times 10 \times 5 \text { (optics) } \\
5 \times 15 \times 20 \text { (electronics) }\end{array}$ & $7 \times 0.1$ \\
\hline IR Mapping Spectrometer (IMS) & 12 & $15 / 1$ & $\begin{array}{l}37 \times 39 \times 83 \text { (optics) } \\
20 \times 25 \times 13 \text { (electronics) }\end{array}$ & $34 \times 0.1$ \\
\hline Laser Altimeter (LA) & 12 & $21 / 21$ & $75 \times 60 \times 60$ & $0.05 \times 0.05$ \\
\hline Ice Penetrating Radar (IPR) & 30 & $61 / 5$ & $\begin{array}{l}20 \times 30 \times 20 \text { (electronics) } \\
+ \\
30-\mathrm{m} \text { dipole }+ \\
10-\mathrm{m} \times 2.6 \mathrm{~m} \text { Yagi } \\
(65 \times 25 \times 25 \text { stowed })\end{array}$ & \\
\hline Thermal Imager (TI) & 11 & $1 / 14$ & $29 \times 37 \times 55$ & $17 \times 0.2$ \\
\hline Magnetometer (MAG) & 2 & $1 / 1$ & $\begin{array}{l}2 \times 2 \times 2(2)+ \\
10-\mathrm{m} ? \text { boom }\end{array}$ & \\
\hline Ion and Neutral Mass Spectrometer (INMS) & 10 & $28 / 28$ & $19 \times 23 \times 32$ & \\
\hline MeV Ion Spectrometer (MIS) & 10 & $10 / 10$ & $20 \times 27 \times 36$ & \\
\hline KeV Ion Spectrometer (KIS) & 7 & $11 / 11$ & $20 \times 27 \times 36$ & \\
\hline TOTAL ALL INSTRUMENTS & 107 & $161 / 93$ & & \\
\hline TOTAL ALL INSTRUMENTS + 43\% contingency & 153 & $230 / 133$ & & \\
\hline Additional radiation shielding & 20 & & & \\
\hline \multicolumn{2}{|c|}{ Orbital average with assumed duty cycles without contingency } & 99 & & \\
\hline
\end{tabular}

\section{Spacecraft Description}

The EE spacecraft is a fully redundant, 3-axis stabilized, MMRTG-powered vehicle carrying a complement of 10 body-fixed instruments plus a Ka-band transceiver for precision gravity measurements. The spacecraft communicates with Earth during the science mission through an articulated 3-m, high-gain antenna (HGA), using $\mathrm{X}$-band. The spacecraft configuration (see Figure 3-6) is largely characterized by its two in-line propellant tanks, which are sized to carry slightly more than $4100 \mathrm{~kg}$ of fuel and oxidizer (for Europa orbital missions, wet mass is approximately 50 to $60 \%$ of launch mass capability). The overall height of the spacecraft is $6.1 \mathrm{~m}$, not including appendages. While it is constrained to not exceed the inside diameter of the launch vehicle fairing, its size on-orbit is dominated by the radar antenna and magnetometer boom in the science configuration (Figure 3-1). The total mass of the spacecraft at launch is $6888 \mathrm{~kg}$ including margin, which yields an unallocated dry mass of $342 \mathrm{~kg}$ with respect to the launch vehicle capability of $7230 \mathrm{~kg}$.

The science instruments are all accommodated on the nadir facing panel to allow for appropriate fields of view for science data taking (see Figure 3-7).

The spacecraft design is leveraged from extensive deep space experience on previous missions, as well as more recent experience from EO and JIMO (see Figure 3-8). The current EE design is not mass, power, or cost optimized, but is centered around science data return. Thus, further tradeoffs are required to be worked with the science community to reach a balance between programmatic, scientific, and engineering constraints.

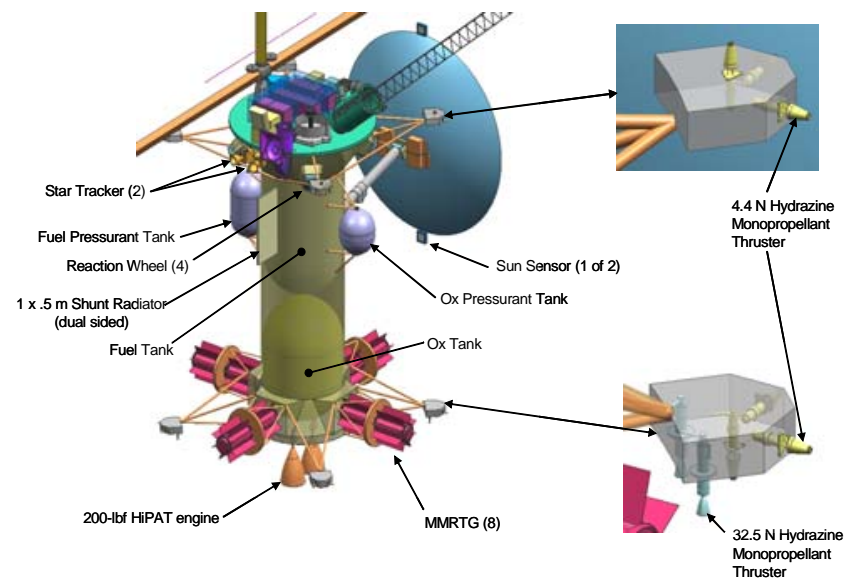

Figure 3-6 Conceptual EE Spacecraft Design

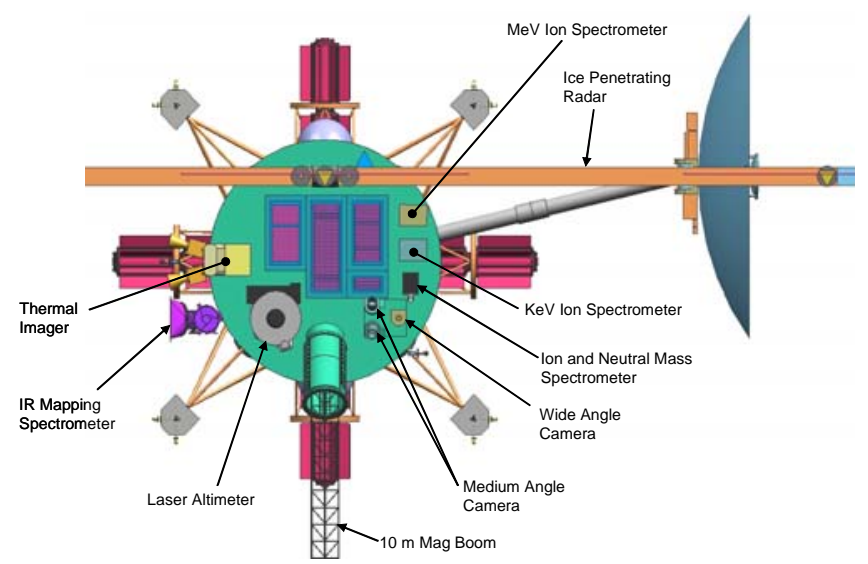

Figure 3-7 Instrument Deck layout 


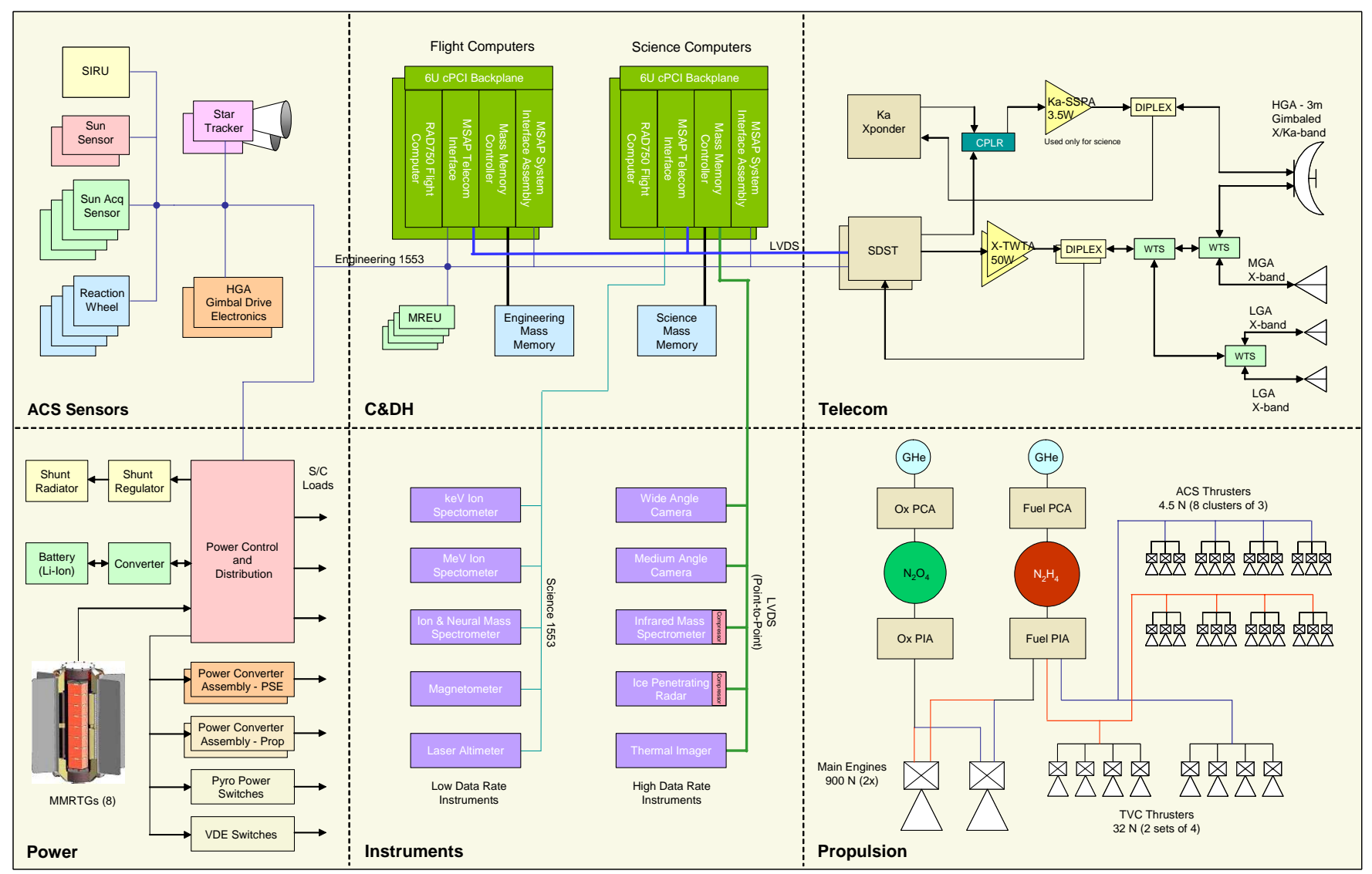

Figure 3-8 EE Spacecraft designs leverages off of extensive deep space experience and more recent Europa studies

\subsection{TECHNICAL DiscusSION}

\section{Science Orbit Operational Scenario}

Once in Europa orbit with a period of approximately 125 minutes, the spacecraft spends a little more than half that time, when it is on the Earth facing side of Europa, in direct communications with Earth and the remaining time occulted from communicating with the Earth. Accordingly, the orbit period is broken into two phases: when there is earth communications ("Comm") and when there is no earth communications ("Non-Comm").

The spacecraft axis of minimum inertia is nominally nadir pointed while in the Europa science orbit. The spacecraft body is further constrained around the roll (nadir-pointed) axis, to maintain the required instrument detector footprint relative to the ground track. Since the HGA is to remain earth-pointed during the unocculted portion of the orbit, and since the spacecraft is rotating 360 degrees per orbit period, the HGA is "despun" using its gimbals. Actually, in the reference design, only one of the gimbals is used to take out the orbital motion, while the other is used to adjust for the slowly changing earthline-to-orbit-plane angle (earth Betaangle). During earth occultations, the antenna is unwound and positioned to the proper direction at the upcoming exit from occultation.
At times, which are dependent on the actual geometry between the HGA and the radar antenna array, the current earth Beta-angle, and the spacecraft position in the orbit, communications will be affected by geometrical interference from the radar antenna array. With proper configuration design, this interference will be centered on the earth occultation period. Geometrical analysis (yet to be performed) will determine whether the period of interference is longer than earth occultation or is contained within the earth occultation period (the design goal). Prelaunch planning will assume that no data is transmitted while there is predicted geometrical interference. The actual geometrical interference will be characterized in flight.

Data are acquired and returned on an orbit-by-orbit basis. Data acquired in one orbit are returned in the same orbit. The mass memory will be essentially empty at the beginning of each Non-Comm phase. Therefore, retransmission of lost data will not be a part of the data return strategy. This is considered acceptable because the demonstrated reliability of the DSN is greater than $95 \%$ at $\mathrm{X}$-Band.

The Comm operating scenario is a new approach which is based in the experiences from but not identical to Cassini, Mars Reconnaissance Orbiter and Magellan. The individual aspects of the approach have been demonstrated though the combination of them has not. 


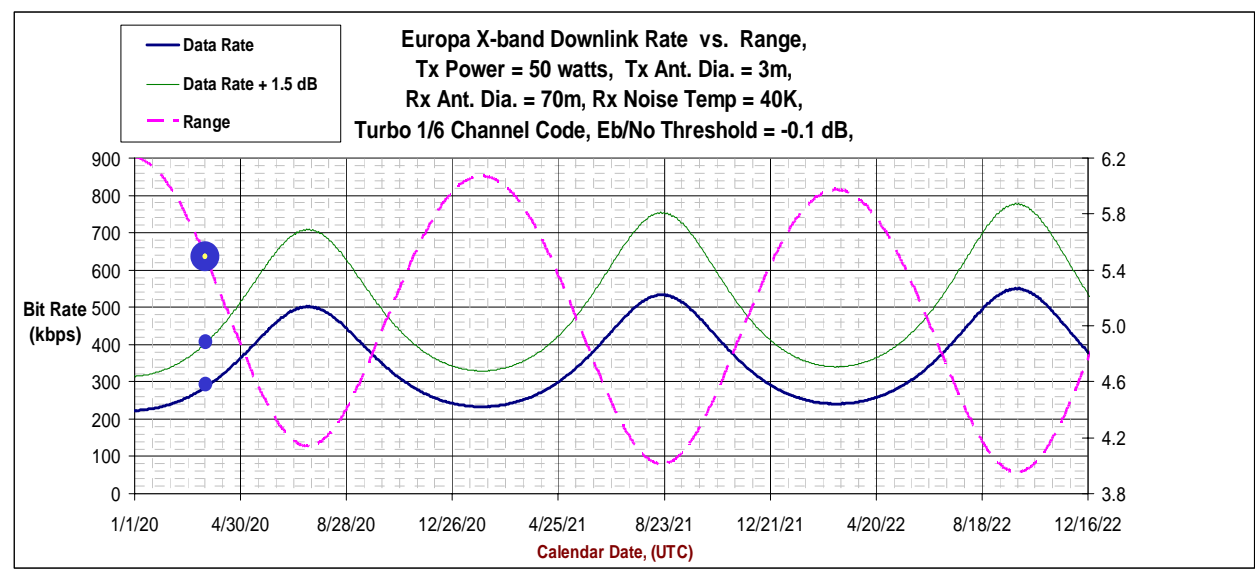

Figure 4-1 Earth-Europa Ranges and data rates as a function of arrival date at Europa

The downlink rate is adjusted in $1 / 2-\mathrm{dB}$ steps to provide the best available downlink rate, taking into account DSN station differences, DSN antenna elevation profile, earth range profile, and Jupiter hot-body noise (during the orbit of Europa around Jupiter, there are times when Jupiter is not in the DSN antenna field of view, which yields better than worst-case performance). These rate adjustments are performed on the spacecraft and at the DSN only while the spacecraft is in earth occultation, and are scheduled in advance, based on telecom performance predictions. With this restriction, there is no reacquisition penalty associated with using multiple data rates in a DSN pass, other than the unavoidable reacquisition penalty associated with each earth occultation.

The hardware and software were designed to meet the derived science data volume requirements at distances of 5.5 AU. As the orbital mission progresses, the spacecraft gets nearer to the Earth and the data rate increases. This study assumed that the spacecraft arrived at Europa at when it is at 5.5 AU and nearing Earth (see Figure 4-1).

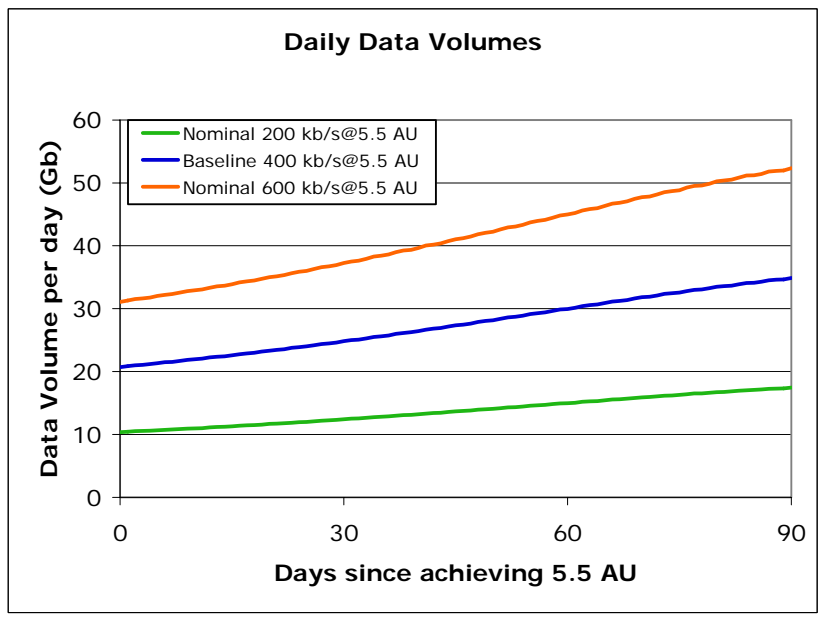

Figure 4-2 Daily Data Volume as a Function of Distance from Earth
By taking advantage of the decreasing Earth-Europa distances, the data rates can increase over the course of the prime mission. The daily average data volume changes over time for the worst case 90 days (spacecraft arrives at Europa at $5.5 \mathrm{AU}$ ) is shown in Figure 4-2 with data rate staring at worst case $5.5 \mathrm{AU}$ and 200, 400, and $600 \mathrm{~kb} / \mathrm{s}$. As shown, the data rates increase by almost a factor of 2 by the end of the 90 day Prime Mission.

Data volume accumulation over the same timeframe is shown in Figure 4-3 using these changing data rates. The exact rates and volumes will be determined by when the spacecraft actually arrives at Europa and what the EuropaEarth geometry is at the time. Both of these analyses assume 24-hour coverage by the DSN for the first 90 days of the mission. The rates are not inherently limited by the hardware or software and robustness in the design allow the mission operations team to take advantage of the increased rates.

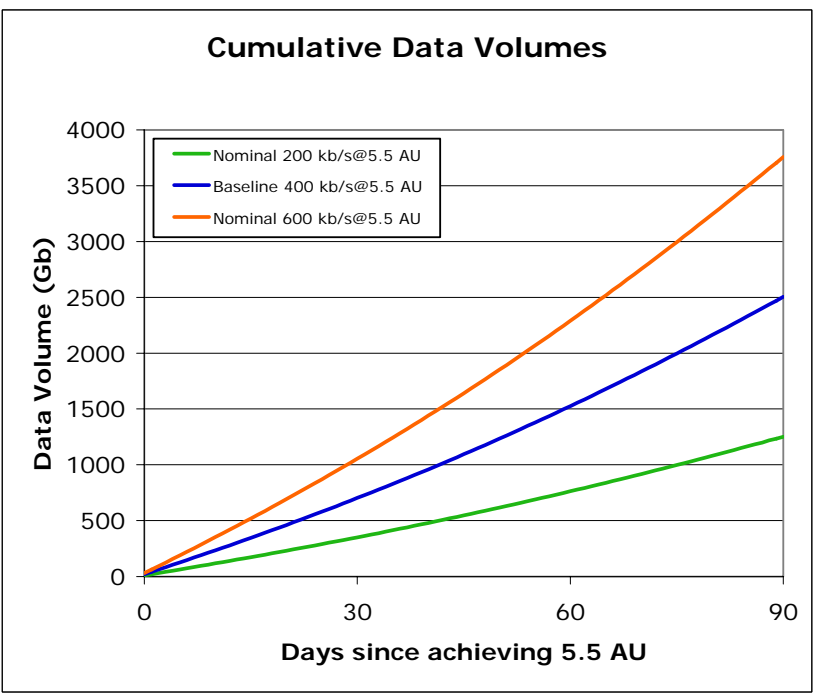

Figure 4-3 Data Volume Accumulation over 90 day accounting for increased data rate at closer distances to Earth 


\section{Data Return Strategy}

For the past decade, solid state mass memories have been used in planetary missions to not only provide intermediate on-board storage of science data but also to allow significant flexibility, operability and cost savings in science data collection. Large mass memories allow very high rate or very large volume data to be collected and can allow it to be down-linked over long periods or complex ground station opportunities. Station coverage gaps or losses of data through faults or noise effects can be recovered through retransmission. Multiple instruments, multiple collection rates, types, on-board data processing and data management can be accommodated. Typical mass memory sizes for recent missions are on the order of $1 / 2$ to several times the daily downlink data volume. For Europa missions like the Europa Explorer under study, 10- to 20-Gbit mass memories would be needed to provide these features. Memories of that size range are not considered feasible in terms of mass and power consumption using parts that can withstand the radiation environment at Europa. A feasible rad-hard memory volume using today's technology can only be about $5 \%$ as large.

From previous studies of missions to Europa, one of the main technology issues identified was always the data storage required to store science data on board the spacecraft. High-density memories such as SDRAM and Flash are not rad-hard, whereas rad-hard memories such as SRAM are not very dense. The availability of rad-hard, high-density memory is still a very significant issue. As a result of the guideline to use only available technologies, a selected implementation was chosen (rad-hard SRAM) for the mass memory which imposed a significant constraint on the mission concept. Thus, this study approached the issue by defining a memory size, which could be achieved within a reasonable mass and power resource level and the mission was designed around that available memory. This resulted in a mission concept built around a mass memory of 300 Mbits dedicated to science data storage. Additional mass memory could be accommodated with increasing mass and power. During Phase A, this approach should be re-evaluated for the then-current state-of-the-state to assess whether the currently adopted approach should be altered for future studies. Investments in rad-hard memories such as CRAM, hard-drives and higher density SRAM and on-board data reduction/selection techniques may open up alternative data return strategies.

The Science Data Return Team was formed to examine methods of reliably returning large volumes of data to Earth with significant constraints on the volume of onboard radiation tolerant mass memory. The team considered architectures and sensitivities of components of the end-toend data return path to determine what viable options existed and what options would be available to trade during future more detailed studies.
Early in the study, the team considered reference architectures and designs from earlier studies and those proposed by the Implementation Assessment Team in its early stages. The components of the end-to-end data return path used by the team for this study include the planning payload, onboard hardware and software data reduction and processing schemes, mass memory, telecommunications, and the DSN. Traditional operations scenarios and constraints were challenged and recommendations were made for design and operations parameters for the orbiter. Trade studies and sensitivity analyses were undertaken to determine the most profitable options for future improvement in the quality and quantity of science data returned.

Several operations scenario recommendations were made to help reduce the science data memory allocation. These include (for the Europa mapping phase):

1) Downlink all data on the orbit collected.

2) Collect data mainly during downlink sessions.

3) Preclude mass memory allocations for data retransmission.

4) Schedule continuous DSN 70-m tracking (or equivalent).

5) Use X-band for highest reliability.

These recommendations were accepted by the study management and they removed all mass memory considerations for data re-transmission, discontinuous DSN coverage, and prioritizing and queuing of data products. Onthe-fly data reduction, compression, processing, packetization and management can still be accommodated and is necessary in some cases. Analysis based on these recommendations showed that mass memory allocations of significantly less than one Gbit could be used while allowing considerable flexibility in data collection among instruments.

Science data acquisition will differ between the two phases, Comma and Non-Comm, due to the limitation of having a small mass memory. Because of the limited memory storage, most of the data acquired during an orbit will be transmitted in near real time. Therefore, instruments that produce large amounts of data at a high-rate will not be operated during Non-Comm phases. Figure 4-4 shows one option for scheduling the science instruments to facilitate the small mass memory.

Data will be acquired from these high-rate instruments in bursts during Comm phases, when the mass memory will be used as a rate buffer between high instrument data rates and the lower downlink rate. Thus the mass memory functions both in a store-for-later-playback mode, and also (primarily) in a rate buffer mode. Data accumulation in the mass memory as a function of the instrument scheduling shown in Figure 4-4 can be seen in Figure 4-5. 


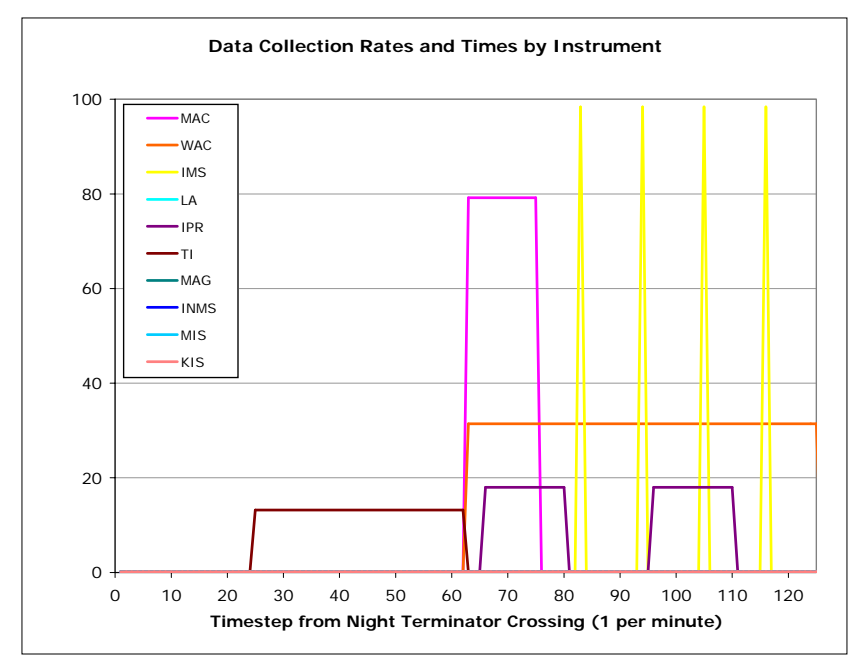

Figure 4-4 Science data collection for a 340kbit/s downlink rate over the course of a full Europa orbit

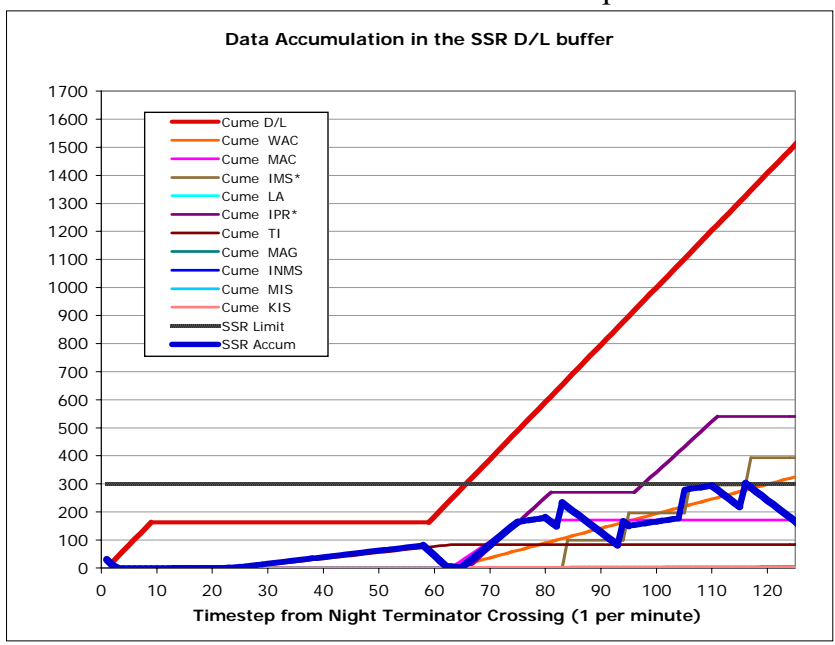

Figure 4-5 Mass memory usage is managed utilizing various science instrument operational scenarios

Because the data return is determined by the communications link, the amount of data returned in Europa orbit is not impacted by limitations on the mass memory although it will limit to some degree the kind of data and the ability to recover lost data. A small mass memory will have more of an impact during the satellite tour when the opportunities to acquire data are brief and there are extended opportunities to transmit high rate data between satellite encounters.

After considering several options, the EE study team selected X-band downlink and DSN 70-m stations as the standard telecom configuration in-order to meet Science data return objectives at an acceptable flight system cost and mission risk. However, since there is currently only one 70-m antenna at each DSN complex, risk is a factor. Many missions mitigate this risk by scheduling critical events during the station overlap periods. However, missions such as $\mathrm{EE}$ that depend on 70-m coverage for longer duration support, must accept the risk, schedule costly back-up 34-m array coverage, or simply lower the data rate for $34-\mathrm{m}$ coverage. Since the reliability of the DSN 70-m support is sufficiently high, (>95\%) the base-lining of 70-m converge was considered appropriate to maximize Science data return. However, several other options should be reassessed as part of any follow-on study or analysis considering the following:

1) The original decision to baseline $X$-band and $70-m$ coverage was made when the Prime Mission baseline was 30 days; it was later increased to 90 days.

2) Even though it offers opportunity for greater downlink data rates, Ka-band was rejected in part since it is affected to a much greater extent than X-band by the weather (attenuation by moisture in the atmosphere). As a result depending on Ka-band was considered too risky for a 30-day Prime Mission where a few days of unusually bad weather over a complex could result in unacceptable coverage gaps. Ka-band for a 9-day Prime Mission might be more acceptable to maximize overall data return.

3) DSN aperture fees for continuous 70-m coverage will be a significant cost factor in any actual proposal (assuming they have to be included in the costing as is usually expected): $\sim \$ 10.3 \mathrm{M}$ for a 30 -day mission, $\sim \$ 31 \mathrm{M}$ for a 90-day mission, in Fiscal Year2006\$.

4) Tying up the 70-m network (at the EE viewing angle) for 90 days could be an issue with NASA due to other conflicting Mission support though the percentage of DSN assets tied up is only $\sim 18 \%$ based on total viewing time per station and total station assets.

5) Although the study assumed 70-m antenna (or equivalent), it is likely that there will be different assets available during the Mission time frame (e.g. array network). In any event, an array of 70-m equivalent will use significant assets, although the array assets will provide more flexibility and reliability.

The data reduction and compression assumptions given with the planning payload are very aggressive. For the reference downlink rate, the requested data compression can be accomplished by the science processor, with a small loss in science data quality, for all but the highest rate instruments. It should be emphasized that instrument Principal Investigators might make very different decisions in adjusting the tradeoff between data quality and amount of compression.

The highest rate instruments in the Planning Payload, the Infrared Mapping Spectrometer (IMS) and the Ice Penetrating Radar (IPR), would need special purpose hardware for internal data reduction because of their very high data collection rates. For medium rate imaging instruments, software image compression is feasible at the reference rates and duty cycles. At significantly higher imaging duty cycles and higher downlink rates, software image compression may begin to strain the limits of the science processor, and hardware image compression may be necessary. Compression for the lowest rate instruments 
(with $100 \%$ duty cycles) can be accomplished in software on the science processor. Mass memory size and downlink volume are not sensitive to compression factors at these very low rates.

Compressed data are more vulnerable to the effects of bit errors such as those produced by Single Event Upsets or communications losses. Compressors for deep space missions should partition data sets into segments that are compressed independently so that the effect of an error or data loss is limited to the affected segment.

Trade studies and sensitivity analyses in the data return context led to several changes from the early point designs. The early Ka-band downlink assumption that was driven by the need for 2-way Ka-band Doppler data and the need for high data rates was changed to a dual band approach using a low power Ka-band transponder for Doppler and ranging data with a moderate power X-band system for data return. The science desire for greater than $300 \mathrm{~kb} / \mathrm{s}$ downlink rates and the operational constraints on the mass memory led to a telecom design characterized by a 50-Wrf TWTA, a 3-m HGA, and a 3-mrad pointing constraint that provides 284 $\mathrm{kb} / \mathrm{s}$ to a 70-m DSN antenna at 5.5 AU and 20 degree antenna elevation angle. Using an operational technique to transmit at the best achievable rate after each orbit occultation, the system takes advantage of increased elevation angles at DSN sites during a tracking pass as well as increasing rates when Europa is farthest away from Jupiter's "hot body" noise temperature. These advantages increase the average data rate to $400 \mathrm{~kb} / \mathrm{s}$ with an orbit-toorbit variation from $220 \mathrm{~kb} / \mathrm{s}$ to $560 \mathrm{~kb} / \mathrm{s}$.

Sensitivity studies were performed to show how science collection and return vary with downlink data rate. Telecom sensitivity studies were performed to show the system cost functions for achieving those increased rates. In the event that future rad-hard mass memory technologies to improve mass memory performance (such as higher density parts), or improved telecom performance (such as DSN array deployment), become available, system design trades can be estimated directly from these sensitivities.

Increases in the science mass memory size can provide more flexibility in science data collection in terms of observation size and timing, but not an increase in the total data returned. Increases in data rate provide opportunities to increase the number of observations and rebalance allocations among the instruments without significantly reducing limits on the maximum size of observations from the high-rate instruments.

Telecommunications design sensitivity curves were generated to evaluate the robustness of the reference design, to show possible ways to increase date rates, and to point to the most useful places to invest resources for those improvements. The reference design occupies a design space where the parameters of mass, power and range can be readily traded off against each other. While variations of factors of 2 to 3 are possible in the design space, higher order improvements would need enhancements in the DSN, such as antenna arrays of hundreds of antennas. In the case of large arrays, the Ka-band should be re-evaluated to avoid bandwidth limitations associated with X-band.

The findings of the data return study conclude that the reference spacecraft design can accommodate the science data collection scenario with a small $300-\mathrm{Mb}$ mass memory allocation. Further, the design will accommodate science data collection scenarios for both lower and higher downlink rates to about a factor of two. The data collection scenarios are highly sensitive to data reduction factors and high burst rate instruments. Future studies should consider additional data collection scenarios to mitigate these issues. Also needed in future studies are analyses to consider the impacts of data format, data accountability, flight-ground interface and operability, and related needs for engineering telemetry.

\section{Science Orbit Selection Strategy}

Analysis of Europa orbiter trajectories with orbit elements in the regime required to satisfy the primary EE science objectives are known to be unstable $[9,10]$. The selected science orbit elements must be compatible with this instability in addition to being appropriate for science desires. The process of selecting appropriate science orbit elements is very preliminary at this time, so the constraints orbit instability imposes on the science orbit elements is uncertain quantitatively. However, the conceptual (qualitative) constraints orbit instability imposes on the science orbit elements can be summarized. The Europa science orbit elements must be constrained to produce an orbit that:

1) Is geometrically satisfactory for required science observations.

2) Is temporally satisfactory for required science observations.

3) Does not present a significant risk of mission loss.

The risk of mission loss is primarily driven by the possibility of temporary loss of control, which precludes orbit maintenance. The most critical loss of control is probably immediately following Europa orbit insertion, but loss of control at other times may be significant.

The temporal quantification of temporary loss of control depends on the details of spacecraft design, and especially the spacecraft fault protection. The fault protection design is far too immature at this time to realistically quantify recovery time to all faults. The time available to regain control immediately following Europa orbit insertion depends on both the orbit insertion errors and the orbit insertion strategy, neither of which is well defined at this time. 
The very preliminary work that has been done on Europa science orbit selection gives confidence that a satisfactory solution is available, but this is unproven. The science orbit used in this study is the science orbit tentatively selected for JIMO.

\section{Design for Radiation}

The radiation design point for any Europa mission is critical for determining the mass required to shield the electronics and materials to function within the environment at Europa (or within the Jovian radiation belts) for the required lifetime. The design point for this study is a Europa 90-day orbital mission with a mean radiation environment. This requires designing for an environment of 3.4 Mrad with a RDF of 1 or $6.8 \mathrm{Mrad}$ with the RDF of 2. Spot shielding to an RDF of 3 is required for parts less than $150 \mathrm{krad}$ hard.
By looking at the accumulated dose based on time in Europa Orbit (Figure 4-6) and probability of actual dose being below design point (Figure 4-7), the design point of 3.4 Mrad (with an RDF of 1) or $6.8 \mathrm{Mrad}$ (with and RDF of 2) was chosen.

his design point gives a very high confidence of lasting 90 days in orbit around Europa while not over-stressing the design as using a higher confidence number would (one or two sigma values) (see Figure 4-8).

The Jupiter radiation model used for this study was the statistical GIRE model [5,6]. The actual design point is 6.8 Mrad, which includes the RDF of 2, which gives approximately $95 \%$ confidence that the actual radiation dose would be below the design point at 90 days and a $50 \%$ confidence that the actual dose would be below the design point at 225 days.

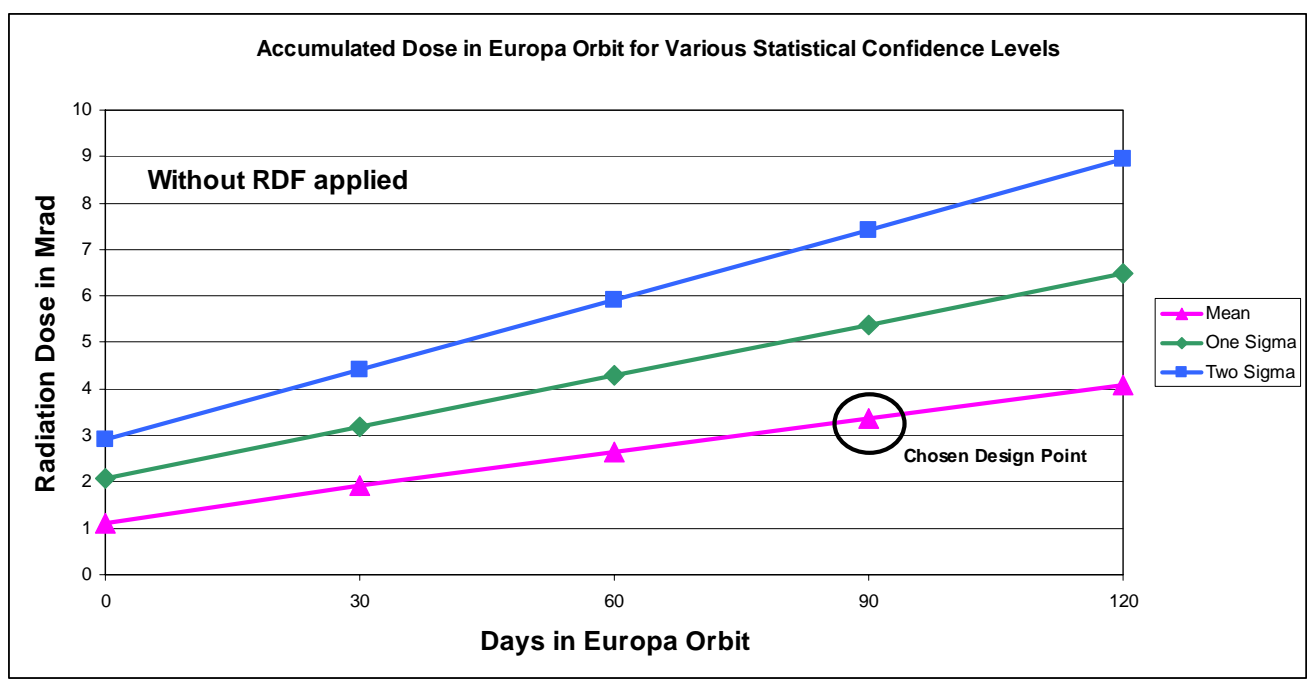

Figure 4-6 Radiation dose (no RDF applied) accumulated over time in Europa Orbit

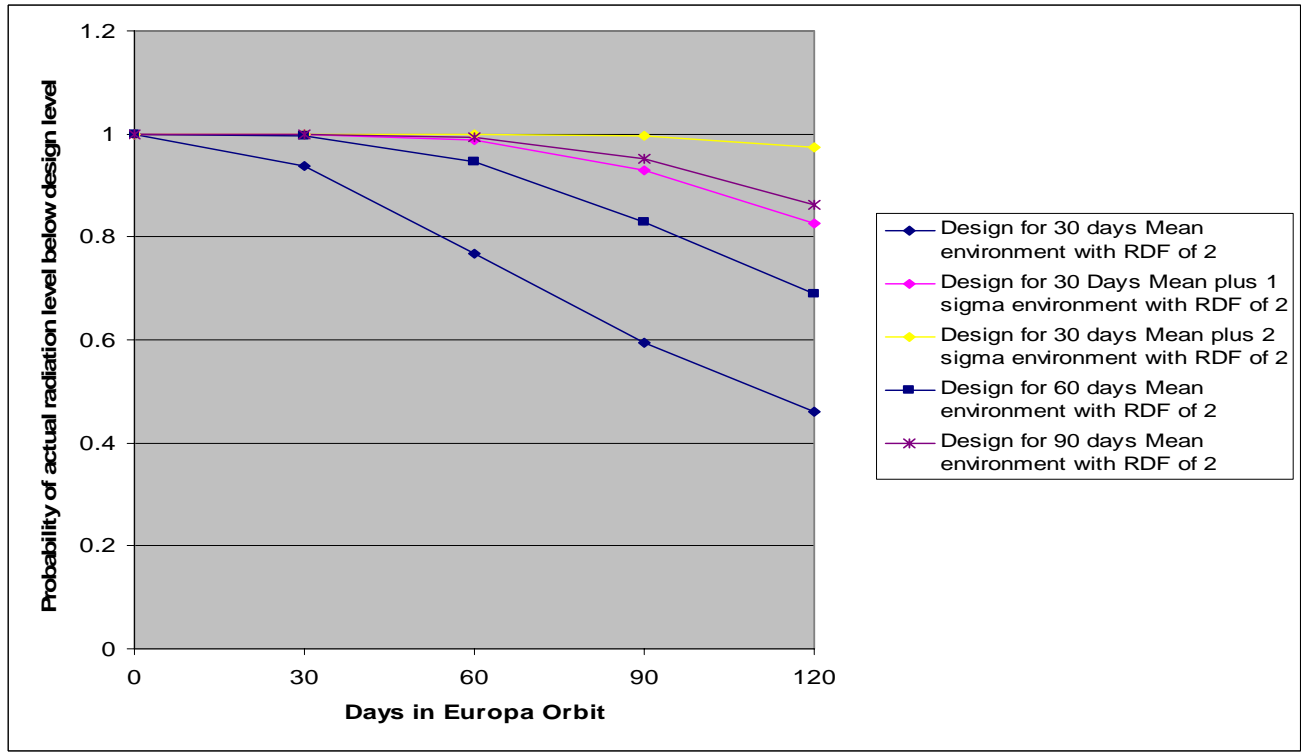

Figure 4-7 Probability that Actual Radiation Level is below Various Design Point Levels 


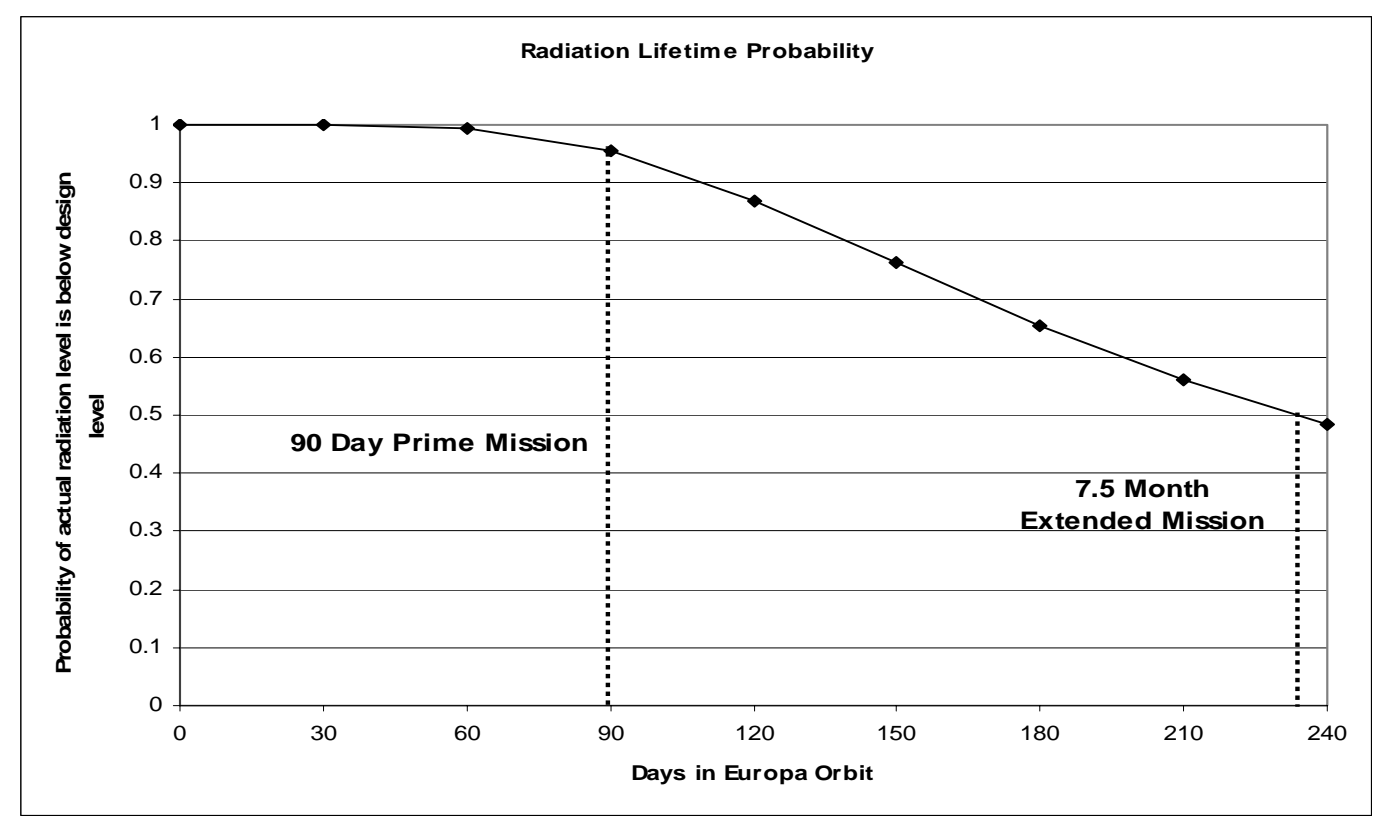

Figure 4-8 Probability that Actual Radiation Environment Seen is Below Selected Design Point

\section{Solar Array Investigation}

A preliminary study was performed to determine the feasibility of using a solar power source in the high radiation environment of Europa to complete the specific science mission as defined by the ESG through the Study Science Team (see Figure 4-9).

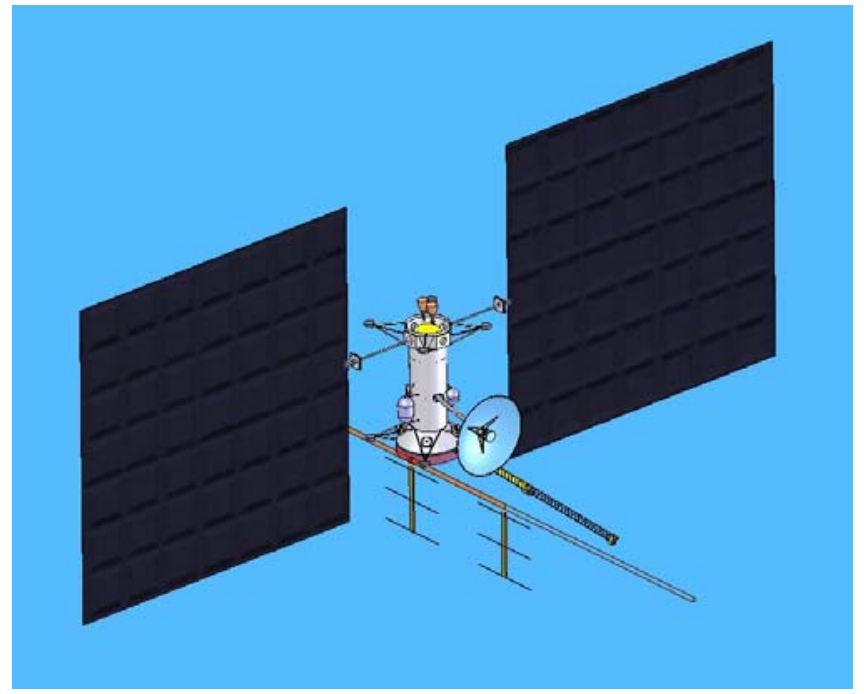

Figure 4-9 Solar Array Concept for Europa Explorer

The technical advancements of solar cells over the past five years relating to both radiation tolerance and Low Intensity Low Temperature (LILT) effects brought about the question of the viability of using solar arrays as a power source for this mission. The Juno mission, a New Frontiers class mission currently in formulation, uses a solar power mission design with current state-of-the-art solar arrays. Juno will enter a high inclination orbit with a perijove well inside the radiation belts and its purpose is to investigate Jupiter; it will not observe the satellites. The Juno mission is spin stabilized and is in a sun-synchronized orbit. It does not have the science pointing requirements required for Europa Explorer science. Unlike EE, which is in solar eclipse approximately $35 \%$ of the time, there are no constraints induced by the solar eclipse portions of the orbit (thermal stability, battery sizing). Finally, with a limited payload and distinct science, telecom and battery re-charge power management modes, orbital average power can be kept low and Juno science can be achieved with a $400-\mathrm{W}$ solar array.

To better understand what would be required for a solar powered Europa Explorer mission, a team was chartered to specifically address the viability of using solar power in Europa orbit to conduct the required science mission. Three main issues were examined:

1) What array size and mass ranges were needed given a set of reasonable spacecraft power assumptions, radiation degradation and state-of-the-art capabilities?

2) Could the spacecraft be stabilized and controlled with these arrays well enough to allow science to meet their objectives?

3) What additional mass and power is required to mechanically integrate and articulate the arrays and to provide the pointing stability required for the science investigations?

See Figure 4-10 for more details. 


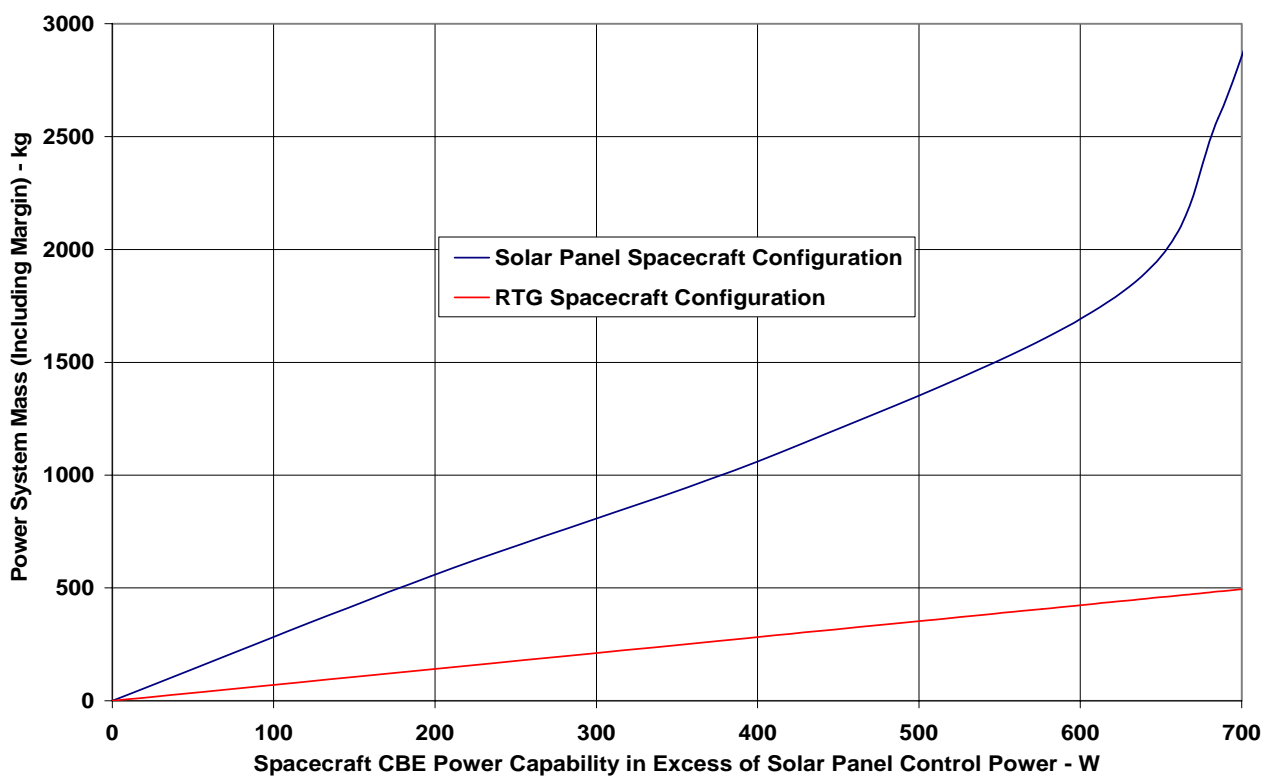

Figure 4-10 Mass/Power Trade off for Solar powered Spacecraft

The study team concluded the required large solar panels are not infeasible per se (absent problems with the low energy heavy ions and atomic oxygen erosion cell degradation), but they create serious spacecraft configuration consequences, especially mechanical accommodations compatible with other spacecraft element requirements and meeting required attitude control and science instrument pointing stability requirements. Infeasibility (like any negative) is very difficult to prove, and this is exacerbated by the complexity of the technical issues, which could not be completely quantified in the relatively short study. However, the large solar panels so intrinsically compromise the attitude control required to satisfy the identified Level-1 Science Requirements (which are stressing even with a more conventional spacecraft) that at best serious science compromise would be required and at worst the mission becomes not worth doing even if the solar power appears nominally adequate.

\section{Optional Design Cases}

In order to understand some of the sensitivities associated with launch vehicles and trajectories, three particular design options were evaluated at a very top level. These options were only briefly explored and no design details were modified to optimize these design options or to assess any additional modifications necessary to accommodate them. A summary of this assessment is shown in Table 4-1.

Table 4-1 Mass Impacts Resulting from Top Level Design Options

\begin{tabular}{|c|c|c|c|c|c|c|c|}
\hline Case & $\begin{array}{l}\text { S/C Dry Mass } \\
\text { (w/ Cont.), kg }\end{array}$ & $\begin{array}{c}\text { Launch Mass } \\
\text { Wet (w/ Cont.) } \\
\text { kg }\end{array}$ & $\begin{array}{c}\mathrm{C3} \\
\mathrm{km} 2 / \mathrm{s} 2\end{array}$ & $\begin{array}{l}\text { LV Injected } \\
\quad \text { Mass } \\
\text { Capability, kg }\end{array}$ & $\begin{array}{l}\text { Unallocated } \\
\text { Mass, kg }\end{array}$ & $\begin{array}{c}\text { Delta from } \\
\text { Reference, } \mathrm{kg}\end{array}$ & Assumptions \\
\hline Reference & 2608 & 6888 & 14.14 & 7230 & 342 & & $\begin{array}{l}\text { Delta IV-H, VEEGA, } \\
8 \text { MMRTGs }\end{array}$ \\
\hline 3 GPHS-RTGs & 2314 & 6565 & 14.14 & 7180 & 615 & 273 & $\begin{array}{l}\text { Delta IV-H, VEEGA, } 3 \text { GPHS- } \\
\text { RTGs and use of acoustic } \\
\text { blankets (that reduce injected } \\
\text { mass capability by } 50 \mathrm{~kg} \text {.) } \\
\end{array}$ \\
\hline DeltaV-EGA & 2373 & 5809 & 26.94 & 5580 & -229 & -571 & $\begin{array}{l}\text { Delta IV-H, DeltaV-EGA, } \\
8 \text { MMRTGs }\end{array}$ \\
\hline Altas V-551 & 2275 & 5227 & 14.14 & 4920 & -307 & -649 & $\begin{array}{l}\text { Atlas V-551, VEEGA, } \\
8 \text { MMRTGs }\end{array}$ \\
\hline $\begin{array}{l}\text { Altas V-551 w/ } \\
3 \text { GPHS-RTGs }\end{array}$ & 1978 & 4930 & 14.14 & 4920 & -10 & -352 & $\begin{array}{l}\text { Atlas V-551, VEEGA, } \\
3 \text { GPHS-RTGs }\end{array}$ \\
\hline
\end{tabular}


There exists a possibility that 3 GPHS-RTGs identical to those flown on Cassini and other missions could be made available for use on this mission. The vibration design level for the GPHS-RTG is lower than that for the MMRTG. Acoustic blankets were required on Cassini to lower the levels seen at the RTG to an acceptable level and would likely be required if used here. This quick study looked at replacing the 8 MMRTGs and replacing them with 3 GPHSRTGs along with the assumed acoustic blankets. This resulted in approximately a $273-\mathrm{kg}$ savings to the reference design with a reduced power margin of $25 \%$ (from $30 \%$ ).

Other indirect trajectories are available and this brief look involved using a $\Delta \mathrm{V}$-EGA trajectory instead of a VEEGA trajectory with a Delta IVH launch vehicle. Using this $\Delta \mathrm{V}-$ EGA trajectory resulted in a launch mass $229 \mathrm{~kg}$ more than the launch vehicle capability.

There is a strong desire to remain dual compatible with the two main Expendable Launch Vehicle manufacturers. If the VEEGA trajectory is used, but the Delta IVH launch vehicle is replaced with an Atlas V-551, the launch mass is $307 \mathrm{~kg}$ greater than the capability of the Atlas V-551.

By combining the Atlas 551 and three GPHS-RTGs for power, the system is just compatible. This combination results on $-10 \mathrm{~kg}$ margin, but reduces the power margin.

\subsection{OPEN ISSUES}

In general, this study output provides a good point design, which can be used as an input to a more detailed study. This study does not presume to be the final answer to a very complex set of trade studies. It merely evaluated the possibility of using current technology to meet a significant set of scientific objectives. During this design study, some key issues were identified which could not be resolved. Most of these (as well as others) should be further explored when funding is available. Many of these issues involve trade offs between mission capability, science return and programmatic constraints, but none appear intractable. Some of the significant remaining open issues are:

\section{Data Return Path Optimization}

There is still a large trade space to optimize the data return path between cost, risk, on-board data storage, antenna size, RF power, DSN coverage and science data collection. This trade will need to be further fleshed out as technology in mass memory advances and other developments progress. Additionally, more in-depth discussions with the DSN are required to understand the implications of the 90-day, 24hour X-band coverage assumption. During a Pre-Phase A and Phase A portion of the mission concept development, decisions on final assumptions will need to be made in conjunction with the Science Definition Team (or equivalent) to settle on a final approach.

\section{Radiation Model}

The Jupiter radiation model used in this study was an update to the initial GIRE model http://www.openchannelsoftware.com/. Further refinements are not only possible but recommended.

1) The method for averaging the Galileo high energy data should be updated to a more accurate logarithmic averaging method instead of using the straight average as is done now.

2) Orbit $\mathrm{C} 22$ and other data imply that about $1 \%$ of the time the jovian radiation environment can exceed the bulk of the data by an order of magnitude. The model should be updated to address the statistical nature of such outlier data and provide a means of estimating their occurrence.

3) The current study made use of a simple geometric shielding factor to account for the effects of Europa on the radiation environment. The detailed interaction of the jovian magnetic field and plasma needs to be evaluated. The result would be used to evaluate the effects of the Europa/jovian interaction on high energy particles.

\section{Spacecraft 3D Modeling}

For the specific point design, the current radiation shield analysis is conservative and includes a total of $233 \mathrm{~kg}$ of shielding (including instrument shielding and uncertainty). The current design point requires approximately $55 \mathrm{~kg}$ for each month of the primary orbital mission around Europa. Spacecraft typically provide 100 to 150 mils aluminum protection when all of the structures, tanks, etc. are incorporated in the model. Variations in shield design incorporating materials changes and layering effects can further decrease a point shield design. Detailed 3D modeling of the spacecraft configuration will be required as the design matures. Variations in the point design will alter the number, size and functionality of electronic boxes which will tend to offset decreases in shielding masses afforded by detailed 3D modeling. This decrease/increase battle will continue as the design evolves over time and the radiation model needs to be built and updated as these modifications occur. Last minute surprises in part/material capabilities will require that a detailed $3 \mathrm{D}$ model be available to assess impacts of late additional shielding requirements.

\section{Radiation Design}

As each layer of further detail gets investigated, issues arise with parts and/or materials, which either are not radiationtolerant or have no data to support their radiation tolerance. The solutions to these issues are best analyzed at the System level to ensure that localized solutions do not undermine the tight resource utilization. During this study, some areas already identified are: propulsion pressure transducers and reaction wheel lubricants. These areas (and others as they are identified) will need to be investigated as the design 
matures. Some options for mitigation include replacement of part or material, spot shielding, placement behind other structure for configurational shielding, functional redundancy, circuit re-design, built in mechanisms for annealing (if useful for the type failure) and operational workarounds in the event of a failure. The system engineering of the radiation design is crucial to the understanding, analysis and mitigation of potential failures.

Effective test methods are critical for understanding the failure modes, analyzing the impacts of the failures is a required to be able to take a systems view of the appropriate set of mitigation actions. Electronic part response to radiation effects cannot always be predicted, nor are all the effects understood. Specifically important to this mission are dose rate effects, impacts of test environment variances from actual environment and the effects of annealing on radiation tolerance (which the Galileo mission design allowed of and EE mission does not). Further investigation into these areas could allow early decisions on electronic part usage, which would reduce impacts of finding issues late in the design cycle.

\section{Design Point Technical Issues}

This study produced only one of many potential point designs, which may be acceptable for this type mission. This particular design point has two identified issues relating to the main engine: center of gravity migration and micrometeoroid protection. The specific issues are not important but each future point design will have its own technical issues related to its specific implementation, which will only be identified, investigated, and resolved with further detailed study.

\section{Lander/Impactor Detailed Concept Study}

The study focused on the feasibility of a spacecraft with onboard instruments orbiting Europa and conducting the prescribed science mission without addressing any lander/impactor mission concepts. If desired, a more detailed assessment of lander and impactor concepts is required given the capabilities and resources available from any Europa mission concept. This work would require both engineering team and science team effort to make sure that the resulting concept is both technically achievable and scientifically desirable. Current concepts are at various levels of fidelity and were conducted with varying sets of requirements and assumptions. A more detailed lander/impactor study should be done to achieve a similar level of confidence in the concept design that fits within the resources and overall framework of a Europa mission concept.

\section{Pu 238 Availability}

The availability of fuel for any RPS is a major issue. NASA and the Department of Energy are working issues related to fuel production, availability and conversion. It is critical that this issue be worked for all but the simplest deep space missions to be viable.

Cost

The focus of this study was on the technical feasibility of meeting a comprehensive set of science objectives within current technology. The mission concept has not been optimized with respect to cost, nor have options with a subset of the science objectives been explored. An extensive amount of effort will be required to fold in cost variables into the design space along with the technical and scientific aspects of the mission design. Complex trades between science return cost and risk will be required to develop mission concepts that are not only scientifically acceptable but also affordable and technically feasible.

\subsection{REFERENCE MISSION DEFINITION}

Subsequent to the initial concept study, trade studies were performed to assess the impact of decreasing the flight system mass to open up the possibility of launching on an Atlas 551 and thus lowering the total mission cost. Options were identified and discussed within the Outer Planets Assessment Group meeting in Tucson in November 2006. A reference mission was adopted which allowed the possibility of launching on an either an Atlas or Delta EELV. This option required some compromise in the mission capability but the science group felt that the resulting mission was still very exciting and robust.

In order to lower the launch mass, several changes were made from the mission concept described in detail earlier:

1) decreasing the complement of science instruments from 10 to 7 (130 kg CBE to $80 \mathrm{CBE} \mathrm{kg}$ ),

2) removing the dedicated science computer (one fully redundant computer system instead of two),

3) lowering of data volume per orbit (from 1.6 Gbits/orbit to 1.2 Gbits/orbit),

1. removing 2 MMRTGs (resulting from lower science, computer and telecom power requirements), and

4) deleting the unallocated mass.

This refined mission concept lowers the launch mass from $6888 \mathrm{~kg}$ to $4920 \mathrm{~kg}$ while still satisfying all the science objectives.

\subsection{CONCLUSIONS}

An orbital mission to Europa has been identified as a high priority by the science community for several years. The difficulty of this type of mission, primarily due to the propulsive capability required and Jupiter's trapped 
radiation, led to many studies that investigated various approaches to meeting the science goals. A flagship-class Europa orbiting mission, which performs a multi-year study of the Jupiter system and meets a vast set of science objectives, can now be developed relying on existing technologies, having significantly more capability and returning considerably more science data than previous conventional propulsion mission concepts. This study has resulted in a mission concept design that returns $22.4-3$ Tbits of data in approximately 90 days around Europa. Cassini will require 2-3 years to return a comparable amount of data from Saturn. During its 3-month (90 day) Prime Mission, the spacecraft would orbit Europa over 1000 times and provide three orders of magnitude more close $(<5000$ $\mathrm{km}$ altitude) Europa observing time than Galileo. A science group was formed to verify that the science objectives and goals were being adequately met by the mission design concept. A planning payload of 10 science instruments was used to evaluate physical, electrical and operational scenarios to ensure that the final design was not only adequate but robust to variations in instrument selection.

The mission concept consists of a single orbiter which traverses to Jupiter by means of a gravity assist trajectory and reaches Jupiter approximately 6 years after launch. The large main engine places the spacecraft into orbit around Jupiter where approximately $1 \frac{1}{2}$ years of Jupiter system science is possible while the spacecraft uses repeated moon gravity assists to lower its orbit until a final burn inserts it into orbit around Europa. Once in Europa orbit, the Prime Mission is 90 days though all identified Priority 1 science measurements can be made in 30 days. This relatively long stay at Europa allows the Science Team to evaluate many flexible science data taking scenarios. Missions operations would be expected to continue well past the 3-month timeframe, potentially up to a year.

At an altitude of approximately $100 \mathrm{~km}$, the spacecraft orbits Europa approximately 12 times in an Earth day. The science payload for the reference mission is a subset of the 10 instruments accommodated in the planning payload and is allocated $80 \mathrm{~kg}(\mathrm{CBE})$ and an orbital average of 75 watts of power (CBE) and a peak power of 140 Watt (CBE). The system is sized to provide a science downlink equating over 17 Gbits per Earth day, which increases as the spacecraft gets closer to Earth. Over the course of 90 days, over 2.4 Tbits of science data can be returned using a continuous downlink strategy which uses $\sim 18 \%$ of the total Deep Space Network (DSN) assets. The science operations are limited by power availability and on-board data storage capability. An exhaustive number of trades will be required to optimize the data collection strategy once the actual instrument and on-board data storage types are selected.

The lifetime of the spacecraft is limited by radiation. The spacecraft will accumulate a total radiation dose of approximately 1.2 Mrad prior to achieving orbit at Europa. Once there, it accumulates approximately $0.72 \mathrm{Mrad}$ for every 30 days in orbit resulting in approximately $3.4 \mathrm{Mrad}$ in the Prime Mission. Thus, the design lifetime involves a trade between mass required to shield the spacecraft and returned science data volume.

This Europa Explorer (EE) concept as described here is made achievable by significant advances in radiation hardened component technologies, now-proven larger launch capabilities and well-established gravity assist trajectory options. The concept relies on traditional chemical propulsion system (similar to Cassini and Galileo), Multi-Mission Radioisotope Thermoelectric Generators (MMRTGs, as employed by Mars Science Laboratory) and a real-time continuous data downlink. The design includes robust design margins based on principles that have been developed over several decades at JPL.

Though no technology developments are required, there are many engineering designs that still require development. Many electronic circuits will require redesign to accommodate radiation hardened parts. Long-lead items such as MMRTGs and propulsion systems need to be initiated very early in the design process to ensure availability for integration.

The challenges and rewards associated with a mission to Europa are captivating both scientifically and technically. The experiences of analyzing the issues, exploring potential solutions, performing technology developments and delving into details have allowed the Jet Propulsion Laboratory (JPL) to provide a robust concept which can out-perform previous radioisotope powered Europa Mission concepts (see Table 6-1).

Continued advancements in areas such as power sources, data storage and DSN capability could further enhance the mission capability but are not mission enabling. Issues related to fuel availability for radioisotope power are crucial and require attention not only for this mission but for a significant number of deep space and flagship class missions. Technical challenges still exist but their nature has changed from the realm of technology development to engineering development.

This paper describes only one mission concept that meets an extensive set of science objectives. There are many outstanding issues related to technical and programmatic items but there appear to be no showstoppers. There exists a much larger trade space in which science objectives need to be balanced within the larger context of programmatic and technical constraints. The existence of an exceptional mission concept using current technology provides the foundation for further investigation and refinement of potential mission concepts. 
Table 6-1 Comparison of Europa Explorer Reference Mission Parameters to Previous Europa Orbiter Concept

\begin{tabular}{|c|c|c|}
\hline Parameter & $\begin{array}{c}\text { Europa Orbiter } \\
\text { EO-2001 }\end{array}$ & $\begin{array}{c}\text { Europa Explorer } \\
\text { EE-2006 } \\
\text { Reference }\end{array}$ \\
\hline Instrument Mass (CBE) & $27 \mathrm{~kg}$ & $80 \mathrm{~kg}$ \\
\hline Instrument Peak Power (CBE) & $27 \mathrm{~W}$ & $140 \mathrm{~W}$ \\
\hline \# Instruments & 4 & 7 \\
\hline Lander & Not Possible & $\sim 1 \frac{\mathrm{kg}}{1 / 2 \text { Years }}$ \\
\hline Prime Mission & Not Practical & 90 Days \\
\hline Jupiter System Science & 30 Days & 2400 Gbits \\
\hline Europa Orbit & 100 Gbits & 135 Days \\
\hline Data Return & & 1200 Gbits \\
\hline (Assumes 24/7 DSN coverage) & 0 Days & \\
\hline Extended Mission & N/A & \\
\hline Europa Orbit & & \\
\hline Data Return & & \\
\hline (Assumes 8/7 DSN coverage) & &
\end{tabular}

\section{REFERENCES}

[1] Solar System Exploration Survey, Space Studies Board, National Research Council, New Frontiers in the Solar System: An Integrated Exploration Strategy, National Academy Press, Washington, D.C., 2003.

[2] Committee on Planetary and Lunar Exploration, National Research Council, A Science Strategy for the Exploration of Europa, National Academy Press, Washington, D.C., 1999.

[3] The Europa Geophysical Explorer Mission Concept Study Final Report (JPL D-32355)

[4] Design, Verification/Validation and Operations Principles for Flight Systems (JPL D-17868)

[5] Galileo Interim Radiation Electron Model, H.B. Garrett, I. Jun, J.M. Ratliff, R.W. Evans, G.A. Clough and R.W. McEntire, 15 September, 2002 (JPL D-24811).

[6] Evans, Robin W., Jupiter Radiation exposure for Europa Orbiter with 1 and 2 Sigma Estimates, IOM 5132-2006-007, February 1, 2006.

[7] Fieseler, P.D.; S.M. Ardalan, and A.R. Frederickson, "The Radiation Effects on Galileo Spacecraft Systems at Jupiter" IEEE Transactions on Nuclear Science 49 (6) 2739-2758 (2002)

[8] Levanas, G. C. "Galileo Mission Inflight Hardware Failure Summary" JPL PD-1625-600 (2003)

[9] Orbit Mechanics About Planetary Satellites Including Higher Order Gravity Fields, M. E. Paskowitz and D. J. Sheeres, AAS 05-190

[10] Transient Behavior of Planetary Satellite Orbiters, M.

E. Paskowitz and D. J. Sheeres, AAS 05-358

\section{BIOGRAPHY}

Karla Clark received her bachelor of science degree in

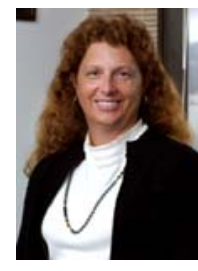
chemical engineering from Rice University in Houston, Texas in 1983. After graduation, Ms. Clark worked at Hughes Aircraft Company developing flight batteries for their communications satellites. Ms. Clark continued her education and received master's degrees in both mechanical engineering (in 1986) and engineering management (in 1988) from the University of Southern California. In 1987 Ms. Clark joined JPL, where she has served as: Task Manager for the Flight Battery Research Technology Objectives and Plans (RTOP) Program; Power Subsystem Engineer and Technical Manager for the Cassini mission; Power Electronics Engineering Group Supervisor, Project Engineer for the Ice and Fire Pre-projects and the Outer Planets / Solar Probe Project; Flight System Manager for the Europa Orbiter; Spacecraft Manager for the Prometheus Project and now the Europa Explorer Study lead.

\section{ACKNOWLEDGEMENTS}

The research described in this paper was carried out by the Jet Propulsion Laboratory, California Institute of Technology, under a contract with the National Aeronautics and Space Administration. 